\title{
Combinatorial $G \times G \times E$ CRISPR screening and functional analysis highlights SLC25A39 in mitochondrial GSH transport
}

\author{
Xiaojian Shi ${ }^{1,5}$, Bryn Reinstadler ${ }^{2,3,4,5}$, Hardik Shah ${ }^{2,3,4}$, Tsz-Leung To ${ }^{2,3,4}$, Katie Byrne ${ }^{1}$, \\ Luanna Summer ${ }^{1}$, Sarah E. Calvo ${ }^{2,3,4}$, Olga Goldberger ${ }^{2,3}$, John G. Doench ${ }^{4}$, Vamsi K. \\ Mootha $^{2,3,4}$, Hongying Shen ${ }^{*}$ \\ ${ }^{1}$ Cellular and Molecular Physiology Department, Yale School of Medicine, New Haven, CT \\ 06511, USA; Systems Biology Institute, Yale West Campus, West Haven, CT 06516, USA \\ ${ }^{2}$ Howard Hughes Medical Institute and Department of Molecular Biology, Massachusetts \\ General Hospital, Boston, MA 02114, USA \\ ${ }^{3}$ Department of Systems Biology, Harvard Medical School, Boston, MA 02115, USA \\ ${ }^{4}$ Broad Institute, Cambridge, MA 02141, USA \\ 5 These authors contributed equally to this work \\ *correspondence: hongying.shen@yale.edu (H.S.)
}

\begin{abstract}
The SLC25 carrier family consists of 53 transporters that shuttle nutrients and co-factors across mitochondrial membranes ${ }^{1-3}$. The family is highly redundant and their transport activities coupled to metabolic state. Here, we introduce a pooled, dual CRISPR screening strategy that knocks out pairs of transporters in four metabolic states - glucose, galactose, OXPHOS inhibition, and absence of pyruvate - designed to unmask the inter-dependence of these genes. In total, we screened 63 genes in four metabolic states, corresponding to 2016 single and pair-wise genetic perturbations. We recovered 19 gene-by-environment $(\mathrm{GxE})$ interactions and 9 gene-by-gene $(\mathrm{GxG})$ interactions. One $\mathrm{GxE}$ interaction hit illustrated that the fitness defect in the mitochondrial folate carrier (SLC25A32) KO cells was genetically buffered in galactose due to a lack of substrate in de novo purine biosynthesis. Another GxE interaction hit revealed non-equivalence of the paralogous ATP/ADP exchangers (ANTs) with ANT2 specifically required during OXPHOS inhibition. GxG analysis highlighted a buffering interaction between the iron transporter SLC25A37 and the poorly characterized SLC25A39. Mitochondrial metabolite profiling, organelle transport assays, and structure-guided mutagenesis suggests SLC25A39 is critical for mitochondrial glutathione (GSH) transport. Our work underscores the importance of systemetically investigating family-wide genetic interactions between mitochondrial transporters across many metabolic environments.
\end{abstract}

\section{Introduction}

The SLC25 family transporters play a critical role in shuttling metabolites into and out of mitochondria ${ }^{1-3}$. The protein family is evolutionarily conserved in nearly all eukaryotes, only missing in a few, highly diverged eukaryotes such as Giardia lamblia and Encephalitozoon cuniculi $^{4}$. Among the 53 SLC25 proteins in human, the most well-characterized ones include adenine nucleotide carriers (ANTs) that exchange ADP for ATP to support cellular bioenergetics and UCP1 that is exclusively expressed in brown adipose tissue and dissipates proton motive force to generate heat. Despite the critical roles, more than 20 members remain transporters of unknown functions. 
Elegant structural characterization of the well-known ANT enable structure-guided characterization of the SLC25 transporters of poorly characterized functions. Each SLC25 transporter is a structural and functional monomer of approximately 300 amino acids with six transmembrane $\alpha$-helices (3-fold pseudo-symmetrical repeats of the two transmembrane helices) surrounding the central cavity. The ANT crystal structures in both cytoplasmic-open (c-state) and matrix-open states (m-state) locked by two inhibitors bound at the solute binding site, carboxyatractyloside and bongkrekic acid respectively, highlighted a conserved alternating-access transport mechanism ${ }^{5}$ enabled by the conformational change upon solute binding at the central cavity $^{6,7}$. The high-level sequence homology and structural similarity among the SLC25 transporters enable both identification of solute-binding residues and prediction of the transporting solute based on the putative chemical groups.

A major challenge for the systematic interrogation of SLC25 transporter functions and their metabolic regulations lies in the high level of redundancy within the family. For instance, 36 members are a part of paralogous subgroups ${ }^{8}$, including four ANT (exchanging ATP for ADP) and four calcium-binding mitochondrial carrier proteins SCaMCs (enabling net adenylate transport). Previously, in our genome-wide CRISPR screen in galactose, a low glucose condition designed to discover genes required for mitochondrial OXPHOS, none of the four ANTs scored as hits ${ }^{9}$. Yet, the ANT inhibitor, bongkrekic acid, induces cell death in low glucose ${ }^{10}$.

Now, inspired by the mitochondria-focused genetic interaction map in Saccharomyces cerevisiae ${ }^{11}$ and the known influence of environment on genetic interactions ${ }^{12}$, we sought a dual genetic perturbation approach to probe the human SLC25 transporters under different metabolic states. We applied a combinatorial CRISPR screening approach to interrogate SLC25 transporters in cellular physiology. By screening in four different media conditions, we uncovered their metabolic regulation, including the fitness defects in the mitochondrial folate transporter SLC25A32 KO cells that can be buffered in galactose condition due to substrate limitation in de novo purine biosynthesis. Strikingly, our screen highlighted a transporter of unknown function, SLC25A39, in which the KO cells' growth defect is buffered by antimycin and the KO exhibits a buffering interaction with the KO of the mitochondrial iron transporter SLC25A37. Follow-up functional studies provide evidence that SLC25A39 is a mitochondrial transporter for the reduced glutathione (GSH).

\section{Results}

We utilized a dual Cas9 enzyme-based knockout strategy ${ }^{13}$ to probe all 53 human SLC25 family members in a pair-wise manner in pooled format. This dual Cas9 system takes advantage of different protospacer adjacent motif (PAM) sequences recognized by Streptococcus pyogenes Cas9 (SpCas9) versus Staphylococcus aureus Cas9 (SaCas9), enabaling simultaneously knocking out two genes (Fig. 1a). To construct the pPapi-SLC25 library, SpCas9 and SaCas9 guides were designed to target 53 SLC25 carriers, seven additional SLC proteins present in MitoCarta 2.0 ${ }^{14}$, BCL2L1, MCL1 (the two paralogous anti-apoptotic proteins) and EEF2 (elongation factor 2, essential for protein translation) as positive controls, 8 non-cutting controls and 19 cutting controls that target olfactory receptors (ORs) as negative controls. This leads to a custom pPapi-SLC25 library of $273 \times 273=74,529$ plasmids for the pooled screen (Fig. 1b). 
We carried out a growth fitness screen in chronic myelogenous leukemia K562 cells across four media conditions (Fig. 1a): (1) standard high glucose media (25 mM glucose, glc), (2) galactose media in which the glucose is replaced by the same concentration of galactose $(25 \mathrm{mM}$ galactose, gal), (3) full media with $100 \mathrm{nM}$ antimycin (antimycin), and (4) full glucose media but lacking pyruvate (-pyruvate). High glucose condition supports cellular bioenergetics using both glycolysis and OXPHOS. Galactose is an epimer of glucose, which only permits mitochondrial OXPHOS ATP production, due to a slower kinetics to utilize galactose in glycolysis ${ }^{15}$. Antimycin is a known inhibitor of the mitochondrial electron transport chain (ETC) complex III inhibiting OXPHOS. Removing pyruvate in the media prevents the recycling of NADH into NAD+ by the cytosolic LDH enzyme, forcing mitochondrial redox shuttle systems to support cytosolic redox balance ${ }^{16}$. All four media conditions are supplemented with uridine and pyruvate (except no pyruvate in pyruvate condition), which are required for the cellular growth under OXPHOS inhibition ${ }^{17}$. In the screen, the cells grew exponentially with expected growth rates: glucose $\sim-$ pyruvate $>$ antimycin $>$ galactose (Fig. 1c). The positive control pair BCL2L1 + MCL1 exhibited the expected synthetic sick genetic interaction as previously reported across all conditions (Fig. 1d) ${ }^{13}$.

We quantified fitness in each condition as the log2-fold-change (LFC) of sgRNA abundance at fifteen days over plasmid DNA (Supplementary Table 1). After applying quality control filters (Supplementary Fig. 1 and Methods), we combined the data from two screening batches, and combined SaCas9 and SpCas9 targeting guides for the same genes. It is worth noting that we only used cutting guides as the negative controls to also control for the deleterious effect during the double-stranded DNA cutting ${ }^{18}$ (see Methods). Together, we scored the genetic perturbation for 63 genes, giving a total of $\left(63^{2}-1 / 2\left(63^{2}-63\right)=\right) 2016$ genetic perturbations, or a total of $(2016 \mathrm{x}$ $4=) 8064$ gene by environment conditions.

We then proceeded to identify three categories of interactions: (i) GxE (Gene x Environment) interaction, wherein a single SLC25 gene KO differentially affects cellular fitness under certain metabolic states; (ii) GxG (Gene x Gene) interaction, wherein the loss of two genes leads to a fitness phenotype that is not the simple additive sum of two single KOs, and (iii) GxGxE (Gene $\mathrm{x}$ Gene $\mathrm{x}$ Environment) interaction, wherein the impact of the pairwise genetic interaction is dependent on the metabolic state.

The results immediately reveal the value of screening pairs of knockouts across different metabolic conditions. Of 53 SLC25A family members, 6 genes scored significantly as essential in at least one of the four media conditions. They include SLC25A1 (citrate transporter), SLC25A26 (SAM transporter), MTCH2 (unknown) in glucose; SLC25A1, SLC25A3 (phosphate transporter), SLC25A19 (thiamine pyrophosphoate transporter), SLC25A26 in galactose; SLC25A1, SLC25A3, SLC25A26 in antimycin; SLC25A1, SLC25A19, SLC25A26, SLC25A32 (folate transporter), MTCH2 (-pyruvate). Importantly, directly comparing the single KO phenotype across four conditions highlighted 19 nutrient-dependent phenotypes that are otherwise mild in any single condition, which we termed GxE interactions (Fig. 2a and Supplementary Table 2). In glucose, 5 pairs of genes exhibited a pairwise $\mathrm{GxG}$ interaction, and $9 \mathrm{GxG}$ interactions scored in at least one of the four conditions, including $8 \mathrm{GxG}$ interactions that are condition-specific. These observations indicate that SLC25 transporters exhibit considerable functional redundancy and that phenotypes can be unmasked only by combinatorial screening under different conditions. 


\section{Gene $x$ Environment (GxE) Interactions}

We quantified the resulting GxE interaction effects by calculating the $Z$-score of the LFC (relative to pDNA) using cutting control guides as the null distribution, which corrects for differences in growth rates between different conditions (Supplementary Table 2). As mentioned above, a total of 19 metabolic state-dependent phenotypes (GxE hits) were discovered (Fig. 2a and Supplementary Table 2). We followed up on two hits that exhibited the strongest GxE interactions, SLC25A19 and SLC25A32, which are transporters of two B family vitamins-derived cofactors, thiamine pyrophosphate (ThPP, or vitamin $\mathrm{B}_{1}$ ) and folate species (vitamin $\mathrm{B}_{9}$ ), respectively.

\section{GxE interactions for SLC25A19, ThPP carrier}

Loss of SLC25A19 results in severe growth defects in glucose, galactose and -pyruvate conditions, which is completely buffered in the antimycin condition in which the respiratory chain is inhibited (Supplementary Fig. 2a). SLC25A19 transports ThPP ${ }^{19}$, the critical cofactor for the E1 subunit of matrix pyruvate dehydrogenase complex (PDC) and oxoglutarate dehydrogenase complex (OGDC) (Supplementary Fig. 2b). Mutations in SLC25A19 cause Amish lethal microcephaly (MCPHA) and thiamine metabolism dysfunction syndrome 4 . We first validated the buffering phenotype in the follow-up studies (Supplementary Fig. 2c). We then re-analyzed our recent genome-wide CRISPR screens performed in the presence of mitochondrial inhibitors, and identified that cell fitness defect in SLC25A19 KOs can be buffered by a spectrum of mitochondrial inhibitors (Supplementary Fig. 2d) ${ }^{20}$, suggesting a direct impairment of OXPHOS function by SLC25A19 loss. Interestingly, the strongest buffering effect is seen with antimycin (Supplementary Fig. 2d). This result, together with the observation that multiple components (OGDH and DLST) and its cofactor modifiers (LIAS and LIPT1) of the OGDC were the top buffering hits with antimycin in the screen ${ }^{20}$, support that the loss of mitochondrial TPP and OGDC might alleviate antimycin-mediated toxicity, a toxicity that has been attributed to the recently reported keto acid dehydrogenase-dependent ROS production ${ }^{21}$.

\section{GxE interactions for SLC25A32, the mitochondrial folate carrier}

The fitness defects of SLC25A32 KOs observed in glucose, antimycin and -pyruvate conditions are buffered in galactose in the screen (Fig. $2 \mathrm{a}$ and $2 \mathrm{~b}$ ). We first confirmed the screen result in the follow-up experiments and showed that two lines of SLC25A32 CRISPR KO cells proliferated slower than the control, but the growth rate of these KOs were comparable to the control cells in the galactose condition (Fig. 2c and Supplementary Fig. 2e).

SLC25A32 is the mitochondrial carrier for the tetrahydrofolate (THF) species in mitochondrial one-carbon (1C) metabolism, as demonstrated by in vitro studies ${ }^{22}$, mouse genetics ${ }^{23}$ and cell culture characterization ${ }^{24-26}$. SLC25A32 was also postulated to transport cofactor $\mathrm{FAD}^{27}$, and its mutations in humans cause riboflavin-responsive exercise intolerance ${ }^{28,29}$. As FAD is the cofactor of the ETC complex II and fatty acid oxidation critical for mitochondrial bioenergetics, a lack of fitness defect of the SLC25A32 KOs in galactose when cells depend on respiration for survival (Fig. 2c and Supplementary Fig. 2e) is surprising, and indicates that either SLC25A32 does not transport FAD into mitochondria in K562 cells, or perhaps that redundant mechanisms exist.

We hypothesized that the observed buffering interaction between the galactose condition and the SLC25A32 $\mathrm{KO}$ is due to their perturbations converge on the de novo purine biosynthesis. SLC25A32 transports THF into mitochondria to support one-carbon (1C) metabolism that 
converts serine into formate, which is then exported into the cytosol for de novo purine biosynthesis (Fig. 2d). In the absence of SLC25A32, cells dramatically accumulated purine intermediates, GAR and AICAR, the substrates of de novo purine biosynthesis enzymes utilizing 10-formyl-THF (Fig. 2e), suggesting perturbation in the pathway. On the other hand, glucose metabolism through pentose phosphate pathway (PPP) generates ribose-5-phosphate (R5P) providing the initial substrate for de novo purine biosynthesis. Replacing glucose with galactose severely depletes de novo purine biosynthesis pathway intermediates in both control and $\mathrm{KO}$ cells (Fig. 2e). Folate species measurement corroborates these results: consistent with a role of SLC25A32 in supporting mitochondrial 1C metabolism, SLC25A32 KOs have significantly reduced cellular 5,10- $\mathrm{me}^{+}$-THF (Fig. 2f), the mitochondrial folate cofactor that is generated by redox-mediated MTHFD2 (Supplementary Fig. 2f). Interestingly, the KO cells also have slightly, albeit not significantly, reduced THF and 5,10-me-THF (Fig. 2f), which may suggest a primary mitochondrial localization of these folate species.

The long-term galactose adaptation might directly inhibit THF cofactor biosynthesis, providing additional means to buffer the defective mitochondrial THF import in the SLC25A32 KOs. Two enzymes involved in cytosolic THF biosynthesis, DHFR and MTHFR, utilize NADPH cofactors (Supplementary Fig. 2f). Long-term galactose adaptation (2 weeks) appeared to reduce the cellular $\mathrm{NADH} / \mathrm{NAD}^{+}$ratio and NADPH/NADP ${ }^{+}$ratio, albeit not significantly (Supplementary Fig. 2h), as well as all major THF species (Supplementary Fig. $2 \mathrm{~g}$ ). The data are consistent with the report that decreased PPP flux and G6PD activity might impair NADPH and THF biosynthesis ${ }^{30}$. The exception is an increase of the mitochondrial 5,10-me $-\mathrm{THF}$, likely due to an upregulation of the mitochondrial $1 \mathrm{C}$ metabolism in galactose ${ }^{31}$.

We further extended the observed buffering interaction by directly inhibiting the folate pathway by either low-folate media or well-known folate pathway inhibitor, methotrexate. Cells cultured in low folate or treated with sub-IC50 concentration of methotrexate proliferated slower than the controls, and this fitness defect is completely blunted while culturing cells in galactose (Fig. $2 \mathrm{~g}$ ). Here, by converting the GxE interaction to an environment-by-environment (ExE) interaction, we show that PPP flux might modulate folate metabolism. In particular, a lower ribose biosynthesis rate might mask folate deficiency or confer methotrexate resistance, with potentially broad implications in metabolic disorders and cancer therapy.

\section{GxGxE interactions in paralogous genes}

To explore pairwise genetic interactions, we scored the strength of the GxG interactions using the $\pi$-score ${ }^{32}$ (Supplementary Fig. 3), which allows comparison of the fitness consequences of a double knockout relative to what is expected by the simple addition of two single knockouts. For genetic perturbations that confer fitness defects, a negative $\pi$-score defines "synthetic sick" and a positive $\pi$-score defines "buffering", respectively (Supplementary Table 3). Scores for all GxG pairs are summarized in Supplementary Figs. 5-8. Here, we focus on SLC25 gene pairs with large effect sizes (absolute $\pi$-score $>0.25$ ) and with significance (FDR $<2 \%$ ). We excluded gene pairs exhibiting "ceiling" and "deader than dead" effects (see Methods).

This procedure for finding genetic interactions highlighted nine SLC25 gene pairs of genetic interaction that scored in at least one media condition (Fig. 3a and Supplementary Table 4). The 
positive control GxG pair (BCL2L + MCL1) and the negative control pair for SLC25 genes that are not expressed (UCP1 and SLC25A31) are shown at the bottom for comparison (Fig. 3a).

Consistent with the notion that SLC25 proteins are highly redundant, we observed strong synthetic sick interactions between 4 paralogous gene pairs $^{8}$ : SLC25A5 x SLC25A6 (ANTs), MTCH1 x MTCH2 (two outer mitochondrial membrane SLC25 proteins of unknown functions involved in mitochondria dynamics ${ }^{33,34}$ ), SLC25A16 x SLC25A42 (CoA carriers) ${ }^{35,36}$, and SLC25A28 x SLC25A37 (iron carriers) ${ }^{37}$.

Among these paralogs, two ANTs, SLC25A5 (ANT2) and SLC25A6 (ANT3), exhibit the strongest synthetic sick interaction in galactose (Supplementary Table 4) and the greatest difference in genetic interactions across conditions (Fig. 3a). ANTs typically import ADP $^{3-}$ in exchange for OXPHOS-produced ATP4- across the inner mitochondrial membrane (Fig. 3c). The human genome encodes four ANTs, and K562 cells express higher level of SLC25A5 (ANT2) and SLC25A6 (ANT3) than SLC25A4 (ANT1), while SLC25A31 (ANT4) is not expressed (Fig. 3d). SLC25A4 is not upregulated in the A5 and A6 DKOs (Fig. 3e), and SLC25A31 cannot be detected in any single or DKOs. In glucose, SLC25A5 $\times$ SLC25A6 DKOs exhibit no fitness defect (Fig. $3 b$ ), however, the same DKO cells exhibited an extreme synthetic sick phenotype in galactose (Fig. $3 \mathrm{~b}$ ), suggesting a redundant but critical role of SLC25A5 and SLC25A6 in supporting OXPHOS when glycolysis is severely inhibited. This redundancy helps to explain why neither carrier scored in our previous "Glu/Gal" screen". A synthetic sick interaction is also observed for the DKO cells in antimycin (Fig. 3b), probably because ANTs run in reverse to import glycolytic ATP4- in exchange with $\mathrm{ADP}^{3-}$ to maintain mitochondrial membrane potential in states of OXPHOS inhibition $^{38,39}$.

Curiously, our screen also highlighted a unique role of SLC25A5. SLC25A5 (ANT2), but not other ANTs, was also a GxE hit, in which single $\mathrm{KO}$ has no phenotype in glucose, but exhibited growth defect in the antimycin condition (Fig. 2a and Fig. 3b). This specific GxE interaction for SLC25A5 was independently supported in our recent mitochondrial inhibitor-sensitized genome-wide CRISPR screens (Fig. 3f) ${ }^{20}$. Interestingly, SLC25A5 is uniquely upregulated during mitochondrial inhibition $^{40-42}$. And, SLC25A5, but not SLC25A6, can restore growth of yeast triple ANT (aac13) mutants in anaerobic conditions ${ }^{40}$. Further study to explore the functional difference between ANTs will be of particular interest.

\section{SLC25A39 KO exhibits both GxG and GxE interactions}

Our screen recovered $\mathrm{GxG}$ interactions between five non-paralogous gene pairs (Fig. 3a). We focused on a poorly characterized SLC25A39 (A39), which exhibits a buffering interaction with the iron carrier SLC25A37 in all four conditions and the stongest interaction in antimycin (Supplementary Fig. 4a), a buffering interaction that we could confirm in the follow-up experiments (Fig. 4a and 4b).

We hypothesized that A39 regulates mitochondrial OXPHOS biology, because A39 is also a GxE screen hit in which the growth fitness defect in the A39 KO cells was buffered in antimycin (Fig. $2 \mathrm{a}$ and Supplementary Fig. 4a). We confirmed the screen result by showing that A39 CRISPR KO cells exhibited a mild growth defect in glucose (Fig. 4c and Supplementary Fig. 4b), which is rescued by ectopically expressing CRISPR-resistant A39 construct (Supplementary Fig. 4c). The 
KO's fitness defect was buffered in antimycin (Fig. 4c) and was enhanced in the galactose condition (supplementary Fig. 4d) consistent with a putative role of A39 in antimycin-sensitive mitochondrial respiration. Indeed, the A39 KO had lower basal and maximum respiration (Fig. 4d), and an increased ECAR due to a compensatory upregulated glycolysis (Fig. 4e). In addition, cellular polar metabolite profiling in A39 KO cells highlighted increased serine and decreased aspartate levels (Fig. 4f and Supplementary Fig. 4f), a metabolic signature of OXPHOS inhibition ${ }^{43-45}$. The decreased respiration, increased glycolysis and cellular metabolic perturbations can be rescued by re-expressing A39 (Supplementary Fig. 4e and Fig. 4f). Together, our initial characterization shows that loss of SLC25A39 impaired mitochondrial respiration, and this helps to explain the observed GxE interaction between SLC25A39 and antimycin in the screen.

SLC25A39 is a transporter of unknown function, but numerous mutant phenotypes have been reported. A39 flies show defects in neurotransmission leading to neurodegeneration in the eyes ${ }^{46}$. Knockdown of A39 in zebrafish leads to profound anemia ${ }^{47}$. Yeast mutant of A39 ortholog $m t m 1 \Delta$ exhibited altered mitochondrial iron homeostasis that could be complemented by the vertebrate A39 sequence ${ }^{47,48}$. A39 resides in a susceptibility locus for epilepsy ${ }^{49}$, and de novo frameshift variant $^{50}$ and damaging missense variants ${ }^{51,52}$ are reported in autism spectrum disorder.

We sought to characterize A39's function by evaluating the impact on mitochondrial metabolism upon knocking out A39. We introduced into suspension K562 cells the previously developed mitoIP construct (3xHA-eGFP-OMP25) and the control construct (3xMyc-eGFP-OMP25) ${ }^{53,54}$. Rapid immunopurification enabled a specific enrichment of mitochondria with little contamination from the ER or lysosome (Supplementary Fig. 4g). Metabolite profiling of the isolated mitochondria revealed a striking depletion of both the reduced and the oxidized glutathione, GSH and GSSG, in the A39 KO mitochondria compared to the controls (Fig. 5a and 5d), a result replicated using an independent guide RNA (Supplementary Fig. 4i). We could also validate this result in KO Hela cells (Fig. 5b and Supplementary Fig. 4h). The GSH/GSSG ratio was not changed (Fig. 5d). For both GSH and GSSG, we did not observe any changes in the whole cell extract (Fig. 5d), implicating a specific regulation in the mitochondrial organelle. Collectively, mitochondrial metabolite profiling shows a decrease both in the reduced and oxidized forms of intramitochondrial glutathione upon A39 loss.

We hypothesized that GSH is the solute that is transported across the mitochondrial membrane by A39. GSH is a tripeptide that is synthesized in the cytosol from three amino acids glutamate, cysteine and glycine (Fig. 5c). To test if GSH is transported in an A39-dependent manner, we designed an organelle-based GSH uptake assay. In the assay, we incubated isolated mitochondria from the control and A39 KO cells with $200 \mu \mathrm{M}$ stable isotope-labeled GSH [M+3] (GSH[glycine-13C2, 15N]), in which two carbons and one nitrogen of the glycine residue are labeled (Fig. 5e). After incubating at room temperature, we washed mitochondria and analyzed them for GSH level by MS. The abundance of labeled GSH is significantly decreased in the KO mitochondria compared to the controls, confirming defective GSH uptake (Fig. 5f and 5g).

Stable isotope analysis of GSSG from this uptake assay further suggests that GSH is indeed imported into the mitochondria and functionally used to synthesize GSSG intramitochondrially. We analyzed the metabolites that are either outside (supernant fraction) or inside mitochondria (washed mitochondrial fraction) from the control mitochondrial uptake assay samples at $30 \mathrm{~min}$ 
time point. In the outside, we only detected doubly labeled GSSG, probably due to spontaneous oxidation of the labeled GSH, and no singly labeled GSSG was detected (Fig. 5h). However, inside of the mitochondria, a significant $13.4 \%$ of GSSG was detected as the singly labeled isotopomer [M+3] (one GSH molecule is labeled), and no doubly labeled GSSG [M+6] (both molecules of GSH are labeled) was detected (Fig. 5h). The most parsimonious explanation for the result is that labeled GSH is imported into the mitochondria and reacts with endogenous, unlabeled intramitochondrial GSH to generate singly labeled GSSG (Fig. 5e). The same GSSG isotopomer patterns were observed in the A39 KO mitochondria uptake samples (Supplementary Fig. 4j), suggesting a similar metabolic fate of the imported $\mathrm{GSH}$ in the $\mathrm{KO}$ mitochondria. And consistent with decreased labeled GSH uptake into the KO mitochondria, singly labeled GSSG isomer [M+3] was decreased in the A39 KO mitochondria (Fig. 5f). Together, the organelle-based GSH uptake assay and stable-isotope analysis support that A39 is required for mitochondrial GSH transport to support intramitochondrial conversion to GSSG.

We next used structural modeling and mutagenesis to gain additional evidence that A39 directly recognizes GSH. The atomic structures of ANTs in the c-state and m-state conformation ${ }^{6,7}$ highlighted a putative solute binding site in the central cavity that is conserved across SLC25 transporters. Previous structure-function analysis on another amino acid ornithine carrier SLC25A15 (ORNT1) identified the critical RE residues (Fig. 6a) at this site for binding C $\alpha$ carboxylate and amino group respectively, two signature chemical groups in all amino acids ${ }^{55}$. Supporting this binding specificity, we found that the corresponding residues were replaced by the KQ residues in the citrate carrier SLC25A1 (TXTP), which retains the positive charged Lys for binding carboxylate group but lacks the negatively charged residue required for amino group binding. We then aligned eukaryotic A39 orthologs sequences spanning diverse taxa including protist Plasmodium species against human SLC25A15 (ORNT1) and SLC25A1 (TXTP) for comparision, and identified two corresponding, highly conserved Arg225 and Asp226 residues (Fig. 6a), supporting A39's amino acid binding. We then modeled the human A39 structure based on the ANT structure c-state conformation and confirmed that the Arg225 and Asp226 residues are indeed facing the central cavity allowing solute access (Fig. 6b). Because GSH contains a $\gamma-$ amide bond preserving the carboxylate and amino group of the glutamate residue of GSH, we hypothesized that the RD residues are critical for A39's binding to the glutamate residue of GSH during its transport (Fig. 6b).

To test this solute recognition mechanism, we expressed either the wild type A39 ${ }^{\mathrm{WT}}$ or alanine mutants (A39 ${ }^{\mathrm{D} 226 \mathrm{~A}}$ single mutant or A39 ${ }^{\mathrm{R} 225 \mathrm{~A} / \mathrm{D} 226 \mathrm{~A}}$ double mutant) into the A39 KO cells. Western blotting the isolated mitochondrial lysates using an anti-A39 antibody demonstrated a depletion of A39 in the KO cells, and the recombinant A39 proteins that migrated at a slightly higher molecular weight (Fig. 6c). The ectopically expressed wild type and mutant A39 proteins are expressed at a level comparable to the endogenous protein in the mitochondria, suggesting that the mutations do not affect protein stability (Fig. 6c). The proliferation defect of the A39 KO cells can only be rescued by the wild type $\mathrm{A} 39$, but not by any solute binding defective mutants, $\mathrm{A} 39^{\mathrm{D} 226 \mathrm{~A}}$ or A39 $225 \mathrm{~A} / \mathrm{D} 226 \mathrm{~A}$ (Fig. 6d). We also tested another A39 ${ }^{\mathrm{K} 329 \mathrm{~A}}$ mutant in which a positively charged residue is highly conserved among all SLC25 transporters (Supplementary Fig. 4k), and the corresponding Arg residue in SLC25A15 (ONRT1) was found not directly involved in solute binding but in mediating solute-induced conformational changes critical for the transport activity $^{55}$. Indeed, this $\mathrm{A} 39^{\mathrm{K} 329 \mathrm{~A}}$ mutant failed to rescue the cell proliferation defect providing 
additional support that conformational change-mediated transport is central to A39 function. To directly evaluate the functional importance of A39's predicted GSH binding, we quantified the impact on mitochondrial GSH level by the amino acid-binding defective mutant A39 ${ }^{\mathrm{D} 226 \mathrm{~A}}$. Using mitochondrial metabolite profiling, the depleted mitochondrial GSH level observed in the A39 KO cells could be rescued by re-expressing A39 ${ }^{\mathrm{WT}}$, but not by the solute-binding defective mutant A39 ${ }^{\mathrm{D} 226 \mathrm{~A}}$ (Fig. 6e). The A39 mutants predicted to be defective in solute binding with no effect on protein stability failed to restore mitochondrial GSH level further supporting the notion that A39 itself is critical for mitochondrial GSH transport. Collectively, these studies indicate that A39 is required for GSH transport into mitochondria.

\section{Discussion}

The study on metabolite translocation across membrane-bound organelles via carrier proteins and its impact on cellular physiology has gained momentum recently in the field of cellular metabolism. In this study, we have generated a combinatorial CRISPR library targeting the human SLC25 transporters, the largest known protein family for mitochondrial metabolite transport, with the goal of exploring pairwise genetic interactions and their dependence on metabolic environment. The functional redundancy and conditional essentiality underscores the need for a combinatorial approach for the SLC25 transporters, as has been demonstrated for other SLC families $^{58}$ and the need to explore different nutrient conditions ${ }^{63,64}$. By screening across four different media conditions, we both recovered known interactions and discovered unanticipated insights into mitochondrial micronutrient and cofactor biology. We found that a defect in mitochondrial folate transport can be buffered by decreasing cytosolic carbohydrate metabolism. We then extended the result to an ExE interaction by showing that the cell fitness defect by methotrexate treatment can be buffered in low glucose conditions - a result predicts that the efficacy of methotrexate in humans may depend on systemic glucose homeostasis and nutritional status. We discuss the screening results and phenotypes for other SLC25 transporters of interest in the Supplementary Notes.

A key finding of the current study is the functional characterization of the mitochondrial transporter SLC25A39. GxGxE screening highlighted SLC25A39 loss as being sensitive to the genetic and environmental background, and follow-up studies indicated mitochondrial respiration was impaired in KO cells. Mitochondrial metabolite profiling, organelle-based uptake assay and structure-function analysis identified a critical role of SLC25A39 in regulating mitochondrial GSH transport. GSH, a tripeptide that is exclusively synthesized in the cytosol by two step enzymes GCLC/GCLM and GSS (Fig. 5c) ${ }^{65}$. Mitochondria lack the GSH biosynthesis pathway and depend on transporters to import GSH. Mitochondrial glutathione, mainly found in the reduced form, represents $10-15 \%$ of total cellular glutathione pool, and yet at a mM concentration similar to that of cytosol ${ }^{65}$.

With a reactive thiol, mitochondrial GSH is critical to support intramitochondrial antioxidant defense system, redox signaling and cofactor metabolism including iron-sulfur cluster biosynthesis. Our work shows that A39 is required for GSH transport, and the screen further suggests that the impact of impaired mitochondrial GSH transport is sensitive to genetic and environmental states, notably iron metabolism. While this manuscript was in revision, Wang et al. manuscript in Biorxiv ${ }^{66}$ independently reported the characterization of SLC25A39 as a mitochondrial GSH transporter and a secondary defect of iron-sulfur cluster deficiency in cells 
depleted of mitochondrial glutathione. This observation might explain the decreased mitochondrial respiration in the A39 $\mathrm{KO}$ cells we have observed and the buffering interaction with the iron transporter SLC25A37 in our screen. Together, our work and that of Wang et al. independently establish a role of SLC25A39 in mitochondrial GSH import, enabling new hypothesis for future basic science and translational studies with implications for a host of human diseases ${ }^{48,47,46,61}$.

\section{Acknowledgements}

We thank Yale School Medicine, Yale West Campus, Systems Biology Institute and Cancer Biology Institute for the instrumentation supports; Li Chen, Xincheng Wu and Josh Rabinowitz laboratory for advice on folate species detection; Broad Genetic Perturbation Platform for assisting the CRISPR screen; Jie Liu for technical support; Guangwei Du for advice on mitochondrial isolation; and members of the Shen lab and Mootha lab, and colleagues at Yale School of Medicine and Yale West Campus for fruitful discussions and feedback. This work was supported by Yale School of Medicine startup fund, NIH grants K99/R00 GM124296 to H.S., R35GM122455 to V.K.M. V.K.M. is an Investigator of the Howard Hughes Medical Institute.

\section{Author contributions}

H.Shen and V.K.M. conceived of combinatorial CRISPR genetic screening. J.G.D. designed the CRISPR library; H. Shen performed the CRISPR screen with the help from T.L.T.; B.R. performed computational analysis of the CRISPR screening results; S.E.C. aided in bioinformatics analysis; O.G. provided technical assistance; H. Shah and H.Shen performed the metabolite profiling for the folates and nucleotides; H. Shen, X.S., L.S., and K.B. performed functional and metabolite analysis of A39 KO mitochondria and cells. V.K.M. supervised the combinatorial CRISPR genetic screening. H. Shen supervised the combinatorial CRISPR genetic screening and the functional follow-up studies; H.Shen. and B.R. wrote the initial manuscript with input from X.S. V.K.M. edited the manuscript. All authors reviewed and approved the manuscript.

\section{Disclosures}

VKM serves on the SAB and receives equity from Janssen Pharmaceutics and 5AM Ventures. JGD consults for Foghorn Therapeutics, Maze Therapeutics, Merck, Agios, and Pfizer; JGD consults for and has equity in Tango Therapeutics.

1 Palmieri, F. The mitochondrial transporter family SLC25: identification, properties and physiopathology. Mol Aspects Med 34, 465-484, doi:10.1016/j.mam.2012.05.005 (2013).

2 Ruprecht, J. J. et al. The Molecular Mechanism of Transport by the Mitochondrial ADP/ATP Carrier. Cell 176, 435-447 e415, doi:10.1016/j.cell.2018.11.025 (2019).

3 Cunningham, C. N. \& Rutter, J. 20,000 picometers under the OMM: diving into the vastness of mitochondrial metabolite transport. EMBO Rep 21, e50071, doi:10.15252/embr.202050071 (2020).

4 Tsaousis, A. D. et al. A novel route for ATP acquisition by the remnant mitochondria of Encephalitozoon cuniculi. Nature 453, 553-556, doi:10.1038/nature06903 (2008).

5 Ruprecht, J. J. et al. Structures of yeast mitochondrial ADP/ATP carriers support a domain-based alternating-access transport mechanism. Proc Natl Acad Sci U S A 111, E426-434, doi:10.1073/pnas.1320692111 (2014).

6 Ruprecht, J. J. et al. The Molecular Mechanism of Transport by the Mitochondrial ADP/ATP Carrier. Cell 176, 435-447.e415, doi:10.1016/j.cell.2018.11.025 (2019). 
7 Pebay-Peyroula, E. et al. Structure of mitochondrial ADP/ATP carrier in complex with carboxyatractyloside. Nature 426, 39-44, doi:10.1038/nature02056 (2003).

8 Palmieri, F. \& Monne, M. Discoveries, metabolic roles and diseases of mitochondrial carriers: A review. Biochim Biophys Acta 1863, 2362-2378, doi:10.1016/j.bbamcr.2016.03.007 (2016).

9 Arroyo, J. D. et al. A Genome-wide CRISPR Death Screen Identifies Genes Essential for Oxidative Phosphorylation. Cell Metab 24, 875-885, doi:10.1016/j.cmet.2016.08.017 (2016).

10 Kano, A., Iwasaki, T. \& Shindo, M. Bongkrekic acid facilitates glycolysis in cultured cells and induces cell death under low glucose conditions. Biochem Biophys Rep 20, 100683, doi:10.1016/j.bbrep.2019.100683 (2019).

11 Hoppins, S. et al. A mitochondrial-focused genetic interaction map reveals a scaffold-like complex required for inner membrane organization in mitochondria. J Cell Biol 195, 323340, doi:10.1083/jcb.201107053 (2011).

12 Zhu, C. T., Ingelmo, P. \& Rand, D. M. GxGxE for lifespan in Drosophila: mitochondrial, nuclear, and dietary interactions that modify longevity. PLoS Genet 10, e1004354, doi:10.1371/journal.pgen.1004354 (2014).

13 Najm, F. J. et al. Orthologous CRISPR-Cas9 enzymes for combinatorial genetic screens. Nat Biotechnol 36, 179-189, doi:10.1038/nbt.4048 (2018).

14 Calvo, S. E., Clauser, K. R. \& Mootha, V. K. MitoCarta2.0: an updated inventory of mammalian mitochondrial proteins. Nucleic Acids Res 44, D1251-1257, doi:10.1093/nar/gkv1003 (2016).

15 Reitzer, L. J., Wice, B. M. \& Kennell, D. Evidence that glutamine, not sugar, is the major energy source for cultured HeLa cells. J Biol Chem 254, 2669-2676 (1979).

16 Titov, D. V. et al. Complementation of mitochondrial electron transport chain by manipulation of the NAD+/NADH ratio. Science 352, 231-235, doi:10.1126/science.aad4017 (2016).

17 King, M. P. \& Attardi, G. Human cells lacking mtDNA: repopulation with exogenous mitochondria by complementation. Science 246, 500-503, doi:10.1126/science. 2814477 (1989).

18 Aguirre, A. J. et al. Genomic Copy Number Dictates a Gene-Independent Cell Response to CRISPR/Cas9 Targeting. Cancer Discov 6, 914-929, doi:10.1158/2159-8290.CD-160154 (2016).

19 Lindhurst, M. J. et al. Knockout of Slc25a19 causes mitochondrial thiamine pyrophosphate depletion, embryonic lethality, CNS malformations, and anemia. Proc Natl Acad Sci U S A 103, 15927-15932, doi:10.1073/pnas.0607661103 (2006).

20 To, T. L. et al. A Compendium of Genetic Modifiers of Mitochondrial Dysfunction Reveals Intra-organelle Buffering. Cell 179, 1222-1238 e1217, doi:10.1016/j.cell.2019.10.032 (2019).

21 Liu, X. et al. Acetate Production from Glucose and Coupling to Mitochondrial Metabolism in Mammals. Cell 175, 502-513 e513, doi:10.1016/j.cell.2018.08.040 (2018).

22 Lawrence, S. A., Hackett, J. C. \& Moran, R. G. Tetrahydrofolate recognition by the mitochondrial folate transporter. J Biol Chem 286, 31480-31489, doi:10.1074/jbc.M111.272187 (2011).

23 Kim, J. et al. Formate rescues neural tube defects caused by mutations in Slc25a32. Proc Natl Acad Sci US A 115, 4690-4695, doi:10.1073/pnas.1800138115 (2018). 
24 McCarthy, E. A., Titus, S. A., Taylor, S. M., Jackson-Cook, C. \& Moran, R. G. A mutation inactivating the mitochondrial inner membrane folate transporter creates a glycine requirement for survival of chinese hamster cells. J Biol Chem 279, 3382933836, doi:10.1074/jbc.M403677200 (2004).

25 Zheng, Y. et al. Mitochondrial One-Carbon Pathway Supports Cytosolic Folate Integrity in Cancer Cells. Cell 175, 1546-1560 e1517, doi:10.1016/j.cell.2018.09.041 (2018).

26 Titus, S. A. \& Moran, R. G. Retrovirally mediated complementation of the glyB phenotype. Cloning of a human gene encoding the carrier for entry of folates into mitochondria. J Biol Chem 275, 36811-36817, doi:10.1074/jbc.M005163200 (2000).

27 Spaan, A. N. et al. Identification of the human mitochondrial FAD transporter and its potential role in multiple acyl-CoA dehydrogenase deficiency. Mol Genet Metab 86, 441447, doi:10.1016/j.ymgme.2005.07.014 (2005).

28 Schiff, M. et al. SLC25A32 Mutations and Riboflavin-Responsive Exercise Intolerance. $N$ Engl J Med 374, 795-797, doi:10.1056/NEJMc1513610 (2016).

29 Hellebrekers, D. et al. Novel SLC25A32 mutation in a patient with a severe neuromuscular phenotype. Eur J Hum Genet 25, 886-888, doi:10.1038/ejhg.2017.62 (2017).

30 Chen, L. et al. NADPH production by the oxidative pentose-phosphate pathway supports folate metabolism. Nat Metab 1, 404-415 (2019).

31 Meiser, J. et al. Serine one-carbon catabolism with formate overflow. Sci Adv $\mathbf{2}$, e1601273, doi:10.1126/sciadv.1601273 (2016).

32 Fischer, B. et al. A map of directional genetic interactions in a metazoan cell. Elife 4, doi:10.7554/eLife.05464 (2015).

33 Zaltsman, Y. et al. MTCH2/MIMP is a major facilitator of tBID recruitment to mitochondria. Nat Cell Biol 12, 553-562, doi:10.1038/ncb2057 (2010).

34 Bahat, A. et al. MTCH2-mediated mitochondrial fusion drives exit from naive pluripotency in embryonic stem cells. Nat Commun 9, 5132, doi:10.1038/s41467-01807519-w (2018).

35 Prohl, C. et al. The yeast mitochondrial carrier Leu5p and its human homologue Graves' disease protein are required for accumulation of coenzyme A in the matrix. Mol Cell Biol 21, 1089-1097, doi:10.1128/MCB.21.4.1089-1097.2001 (2001).

36 Fiermonte, G., Paradies, E., Todisco, S., Marobbio, C. M. \& Palmieri, F. A novel member of solute carrier family 25 (SLC25A42) is a transporter of coenzyme A and adenosine 3',5'-diphosphate in human mitochondria. J Biol Chem 284, 18152-18159, doi:10.1074/jbc.M109.014118 (2009).

37 Shaw, G. C. et al. Mitoferrin is essential for erythroid iron assimilation. Nature 440, 96100, doi:10.1038/nature04512 (2006).

38 Nicholls, D. G. Bioenergetics 4th Edition. (2013).

39 Buchet, K. \& Godinot, C. Functional F1-ATPase essential in maintaining growth and membrane potential of human mitochondrial DNA-depleted rho degrees cells. J Biol Chem 273, 22983-22989, doi:10.1074/jbc.273.36.22983 (1998).

40 Giraud, S., Bonod-Bidaud, C., Wesolowski-Louvel, M. \& Stepien, G. Expression of human ANT2 gene in highly proliferative cells: GRBOX, a new transcriptional element, is involved in the regulation of glycolytic ATP import into mitochondria. J Mol Biol 281, 409-418, doi:10.1006/jmbi.1998.1955 (1998). 
41 Bonod-Bidaud, C. et al. Induction of ANT2 gene expression in liver of patients with mitochondrial DNA depletion. Mitochondrion 1, 217-224 (2001).

42 Kuhl, I. et al. Transcriptomic and proteomic landscape of mitochondrial dysfunction reveals secondary coenzyme Q deficiency in mammals. Elife 6, doi:10.7554/eLife.30952 (2017).

43 Bao, X. R. et al. Mitochondrial dysfunction remodels one-carbon metabolism in human cells. Elife 5, doi:10.7554/eLife.10575 (2016).

44 Birsoy, K. et al. An Essential Role of the Mitochondrial Electron Transport Chain in Cell Proliferation Is to Enable Aspartate Synthesis. Cell 162, 540-551, doi:10.1016/j.cell.2015.07.016 (2015).

45 Sullivan, L. B. et al. Supporting Aspartate Biosynthesis Is an Essential Function of Respiration in Proliferating Cells. Cell 162, 552-563, doi:10.1016/j.cell.2015.07.017 (2015).

46 Slabbaert, J. R. et al. Shawn, the Drosophila Homolog of SLC25A39/40, Is a Mitochondrial Carrier That Promotes Neuronal Survival. J Neurosci 36, 1914-1929, doi:10.1523/jneurosci.3432-15.2016 (2016).

47 Nilsson, R. et al. Discovery of genes essential for heme biosynthesis through large-scale gene expression analysis. Cell Metab 10, 119-130, doi:10.1016/j.cmet.2009.06.012 (2009).

48 Luk, E., Carroll, M., Baker, M. \& Culotta, V. C. Manganese activation of superoxide dismutase 2 in Saccharomyces cerevisiae requires MTM1, a member of the mitochondrial carrier family. Proc Natl Acad Sci U S A 100, 10353-10357, doi:10.1073/pnas.1632471100 (2003).

49 Sirén, A. et al. Suggestive evidence for a new locus for epilepsy with heterogeneous phenotypes on chromosome 17q. Epilepsy Res 88, 65-75, doi:10.1016/j.eplepsyres.2009.09.022 (2010).

50 Iossifov, I. et al. De novo gene disruptions in children on the autistic spectrum. Neuron 74, 285-299, doi:10.1016/j.neuron.2012.04.009 (2012).

51 Krumm, N. et al. Excess of rare, inherited truncating mutations in autism. Nat Genet 47, 582-588, doi:10.1038/ng.3303 (2015).

$52 \mathrm{Li}$, J. et al. Targeted sequencing and functional analysis reveal brain-size-related genes and their networks in autism spectrum disorders. Mol Psychiatry 22, 1282-1290, doi:10.1038/mp.2017.140 (2017).

53 Chen, W. W., Freinkman, E. \& Sabatini, D. M. Rapid immunopurification of mitochondria for metabolite profiling and absolute quantification of matrix metabolites. Nat Protoc 12, 2215-2231, doi:10.1038/nprot.2017.104 (2017).

54 Chen, W. W., Freinkman, E., Wang, T., Birsoy, K. \& Sabatini, D. M. Absolute Quantification of Matrix Metabolites Reveals the Dynamics of Mitochondrial Metabolism. Cell 166, 1324-1337.e1311, doi:10.1016/j.cell.2016.07.040 (2016).

55 Monne, M. et al. Substrate specificity of the two mitochondrial ornithine carriers can be swapped by single mutation in substrate binding site. J Biol Chem 287, 7925-7934, doi:10.1074/jbc.M111.324855 (2012).

56 Kory, N. et al. MCART1/SLC25A51 is required for mitochondrial NAD transport. Sci $A d v$ 6, doi:10.1126/sciadv.abe5310 (2020).

57 Luongo, T. S. et al. SLC25A51 is a mammalian mitochondrial NAD $(+)$ transporter. Nature 588, 174-179, doi:10.1038/s41586-020-2741-7 (2020). 
58 Girardi, E. et al. Epistasis-driven identification of SLC25A51 as a regulator of human mitochondrial NAD import. Nat Commun 11, 6145, doi:10.1038/s41467-020-19871-X (2020).

59 Fiermonte, G. et al. Identification of the mitochondrial glutamate transporter. Bacterial expression, reconstitution, functional characterization, and tissue distribution of two human isoforms. J Biol Chem 277, 19289-19294, doi:10.1074/jbc.M201572200 (2002).

60 Vozza, A. et al. UCP2 transports C4 metabolites out of mitochondria, regulating glucose and glutamine oxidation. Proc Natl Acad Sci U S A 111, 960-965, doi:10.1073/pnas.1317400111 (2014).

61 Yoneshiro, T. et al. BCAA catabolism in brown fat controls energy homeostasis through SLC25A44. Nature 572, 614-619, doi:10.1038/s41586-019-1503-x (2019).

62 Sprenger, H. G. et al. Cellular pyrimidine imbalance triggers mitochondrial DNAdependent innate immunity. Nat Metab, doi:10.1038/s42255-021-00385-9 (2021).

63 Rossiter, N. J. et al. CRISPR screens in physiologic medium reveal conditionally essential genes in human cells. Cell Metab, doi:10.1016/j.cmet.2021.02.005 (2021).

64 Costanzo, M. et al. Environmental robustness of the global yeast genetic interaction network. Science 372, doi:10.1126/science.abf8424 (2021).

65 Mari, M. et al. Mitochondrial glutathione: features, regulation and role in disease. Biochim Biophys Acta 1830, 3317-3328, doi:10.1016/j.bbagen.2012.10.018 (2013).

66 Wang, Y. et al. SLC25A39 is necessary for mitochondrial glutathione import in mammalian cells. bioRxiv, 2021.2009.2015.460381, doi:10.1101/2021.09.15.460381 (2021). 


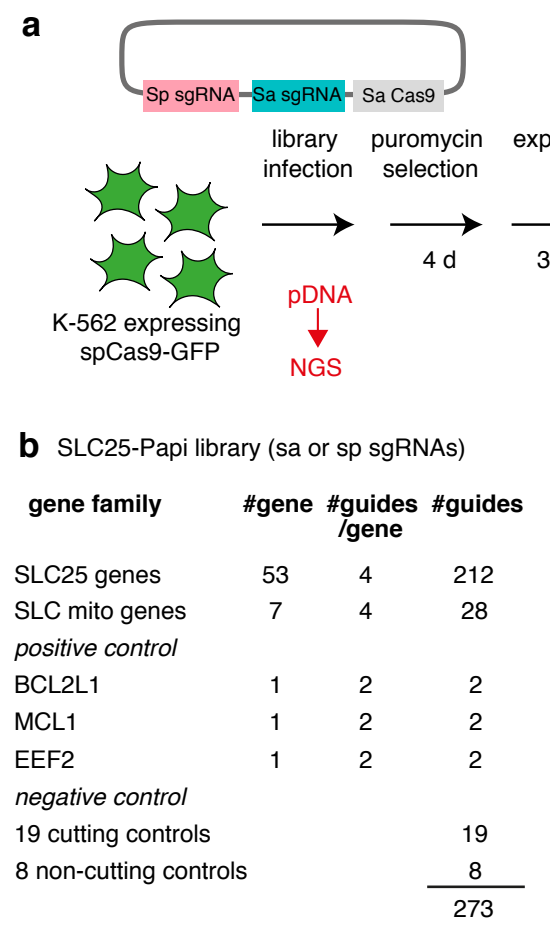

library size $273 \times 273=74,529$

C screen growth rate

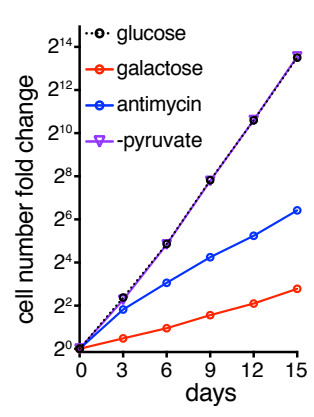

d positive control pairs

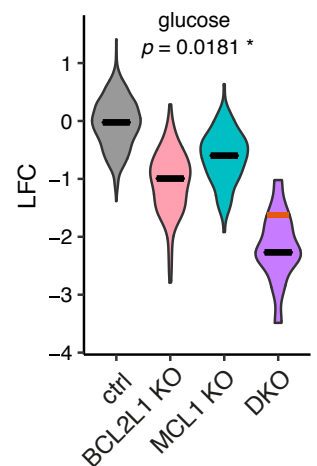

Fig. 1 | Combinatorial CRISPR screen of the SLC25 family transporters across four media conditions. a, Schematic overview of the combinatorial CRISPR strategy. b, SLC25-Papi library construction. c, Growth rate of the pooled CRISPR screen. The batch 2 growth rate was shown and representative of the two batches. d, positive control pair BCL2L1 and MCL1. Data shown are $\log 2$ fold changes from day 15 in the glucose condition. Grey, control-control pair; pink, BCL2L1 x Ctrl or Ctrl x BCL2L1; blue, MCL1 x Ctrl or Ctrl x MCL1; and purple, the double knockouts of BCL2L1 x MCL1 or MCL1xBCL2L1. The black lines indicate the median and the red line indicates the expected value for the double knockout based on the sum of the single knockouts when there is no genetic interaction. 


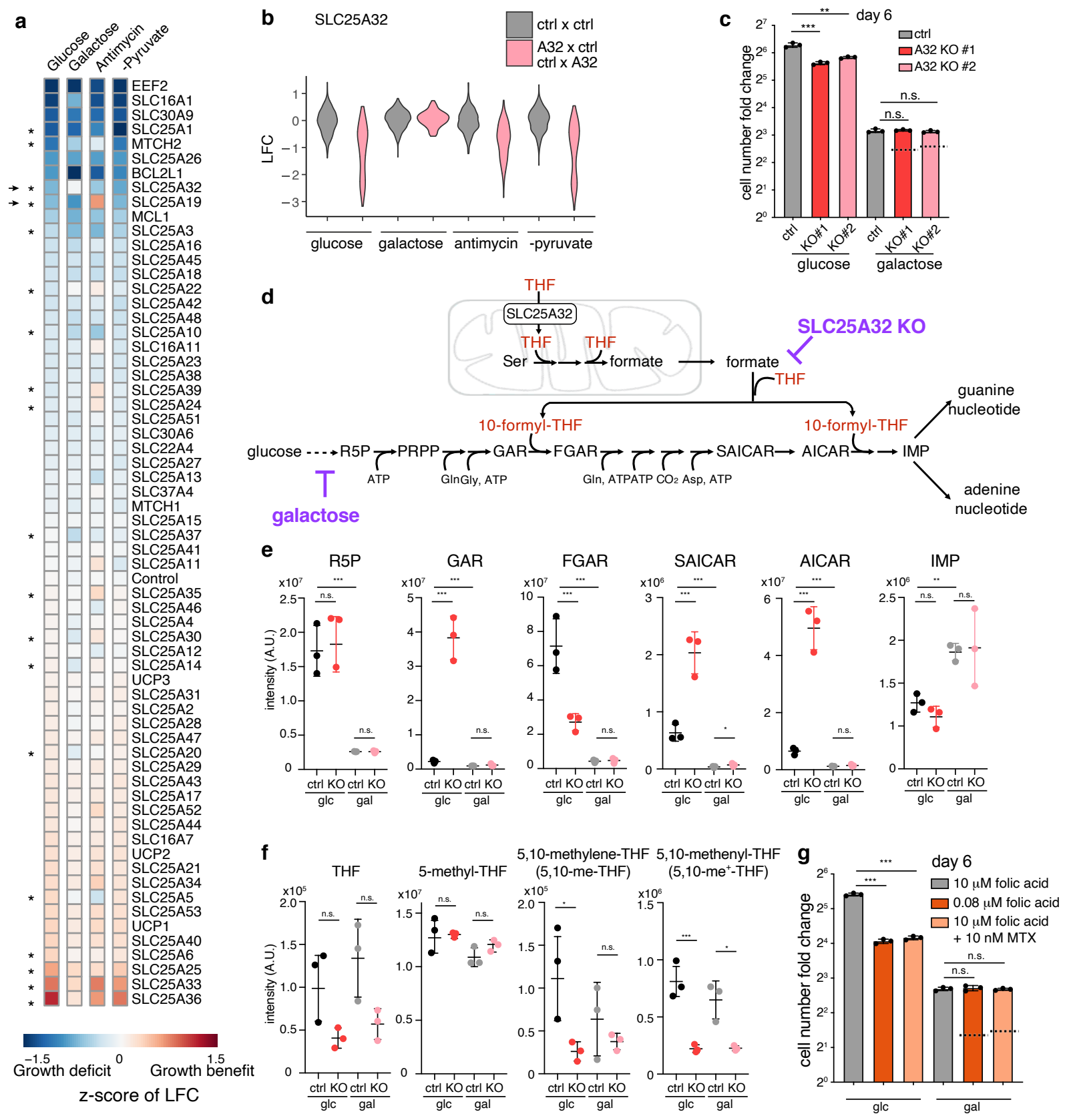

Fig. 2 | Gene $x$ Environment (GxE) mapping indicates that loss of mitochondrial folate metabolism can be buffered in galactose culture conditions. a, A heatmap depicts the fitness of single gene KOs across all four media conditions. Values are $Z$-scores of log fold change (LFC) value of the targeting guide abundance. Asterisks highlight the 19 significant "GxE" interactions, i.e., those knockouts that cause fitness defects differentially across the conditions. Arrows indicate two hits that were experimentally validated and pursued in the current study: SLC25A32 and SLC25A19 (see Supplementary Fig 2). b, SLC25A32 single KO phenotype across four conditions in the screen, showing the buffering effect in galactose. Grey, the controlcontrol distribution; pink, SLC25A32 single KOs ("A32 x Ctrl" or "Ctrl x A32" combined). c, 
Follow-up studies show growth fitness of SLC25A32 CRISPR KO cells generated by two different sgRNAs in glucose and galactose conditions. The dotted line denotes the expected LFC, based on an additive model, if there was no genetic interaction. $\mathbf{d}$, de novo purine biosynthesis pathway. e, LC-MS measurement of purine intermediates in SLC25A32 KO and control cells in glucose and galactose condition for 2 days. f, LC-MS measurement of tetrahydrofolate (THF) cofactors in SLC25A32 KO and control cells in glucose and galactose condition for 2 days. $\mathbf{g}$, Growth fitness of wild type cells either cultured in low folate media $(0.08 \mu \mathrm{M})$ or treated with DHFR inhibitor methotrexate at sub-IC50 dose $(10 \mathrm{nM})$, in glucose and galactose condition. The dotted line denotes the expected LFC, based on an additive model, if there was no genetic interaction. Significance level were indicated as $* * * p<0.001, * * p<0.01, * p<0.05$ and n.s. $p>0.05$. 


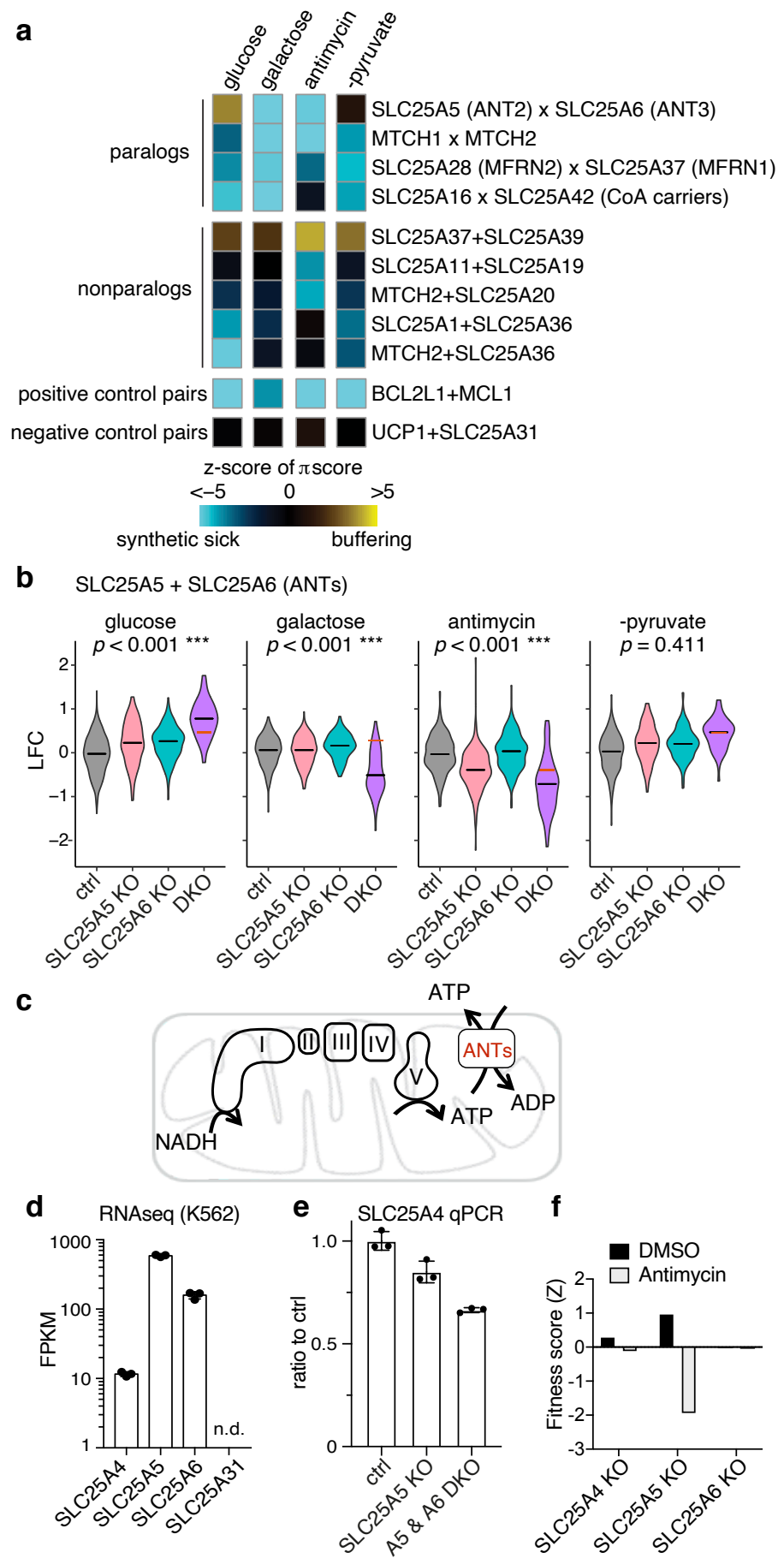

Fig. 3 | Identification of Gene x Gene x Environment (GxGxE) interactions. a, Heatmap shows the $\pi$-score for pairwise genetic interaction. The values are z-transformed to compare across media conditions, as described in the methods. For comparison, a positive control pair (BCL2L1 x MCL1) that are always detrimental when jointly knocked out and another pair (UCP1 x SLC25A31) that do not interact are shown. The rows and columns are not clustered. $\mathbf{b}, \mathrm{LFC}$ values from the screen are shown to illustrate the strong synthetic sick genetic interaction between SLC25A5 and SLC25A6 only in the galactose and antimycin conditions, but not in glucose or -pyruvate condition. The red line indicates the expected LFC, based on an 
additive model, if there were no genetic interaction. The black line indicates the actual median LFC value. c, The cartoon illustrates the function of ANTs in supporting bioenergetics. d, Transcript levels of the four ANTs in K562 cells (data plotted from the published PBS vehicle treated K562 cells RNA-seq dataset GSE74999). e, qPCR of SLC25A4 mRNA level in SLC25A5 KO and SLC25A5 and SLC25A6 DKO cells. f, Re-analysis of the ETC inhibitordependent genome-wide CRISPR screens showed SLC25A5 KO leads to a selective fitness defect in antimycin condition. 

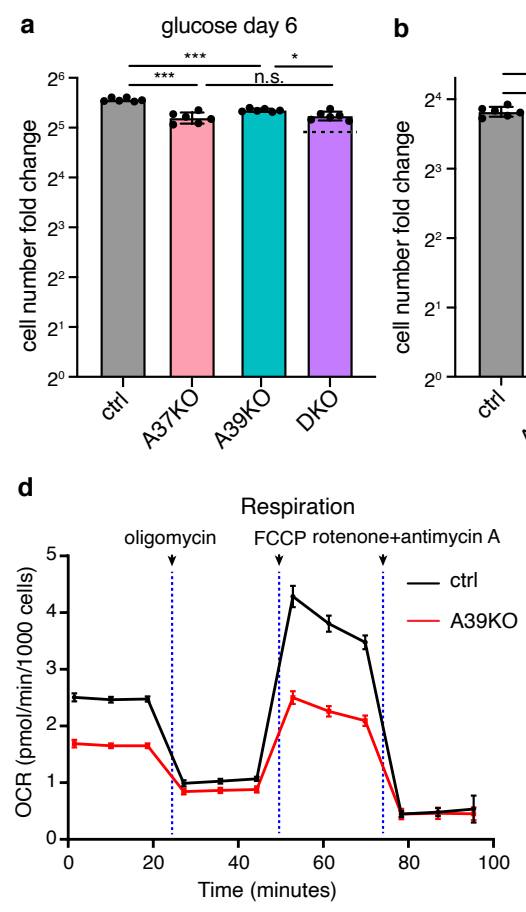
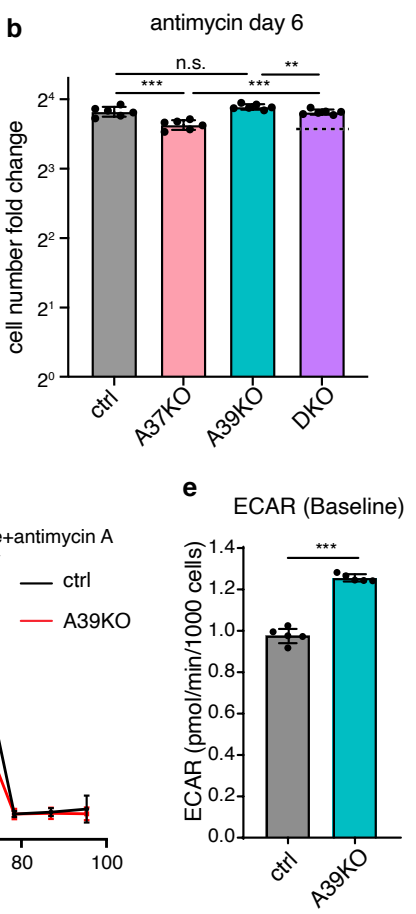
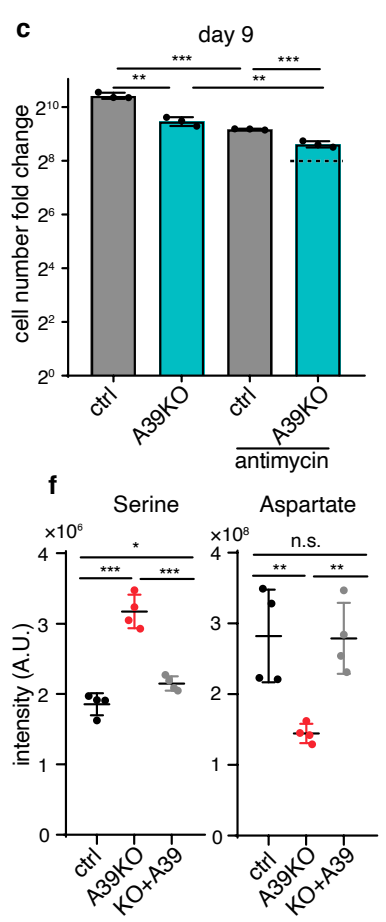

Fig. 4 | The poorly characterized carrier SLC25A39 (A39) is critical for mitochondrial respiration. a, b, Validation of the genetic buffering interaction between SLC25A37 and SLC25A39 loss both in the glucose condition and the antimycin condition. The dotted line indicates the expected cell number, based on an additive model, if there were no genetic interaction. c, Growth fitness defect in A39 CRISPR KO cells, which is buffered in the antimycin condition. The dotted line indicates the expected cell number, based on an additive model, if there were no specific genetic interaction between A39 KO and antimycin. d, Oxygen consumption rate (OCR) for A39 KO cells during Seahorse mito stress test. e, The basal extracellular acidification rate (ECAR) for A39 KO cells. f, LC-MS measurement of serine and aspartate in control, $\mathrm{A} 39 \mathrm{KO}$ and the rescued cells. 


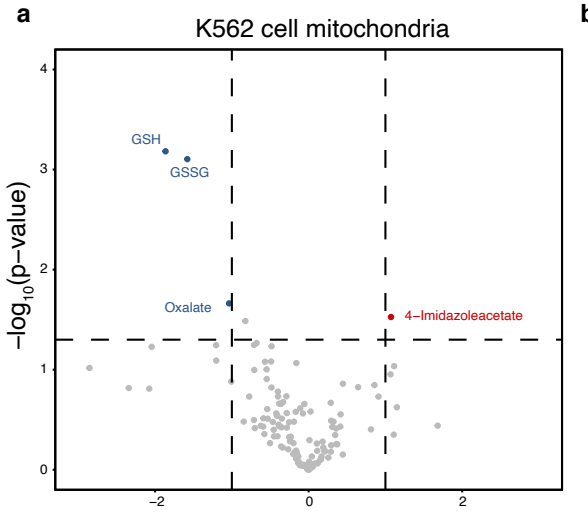

$\log _{2}$ Fold Change (SLC25A39 KO/ctrl)

c
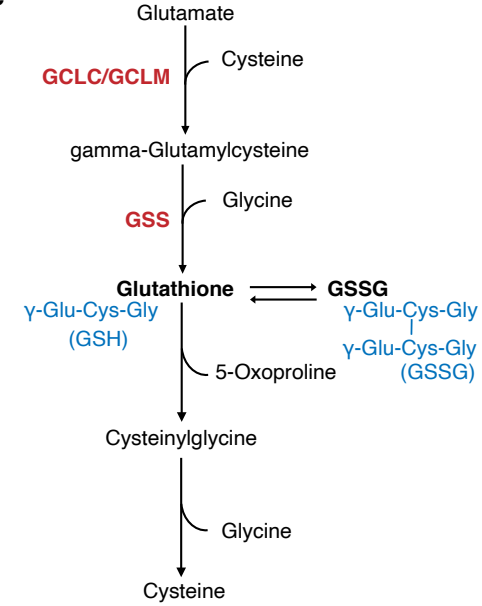

e

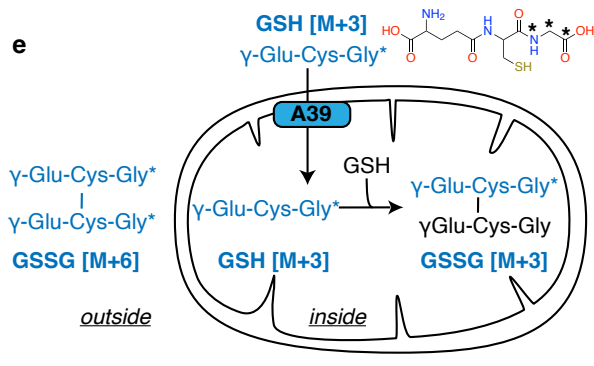

g

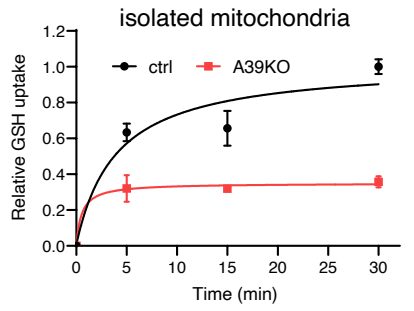

b

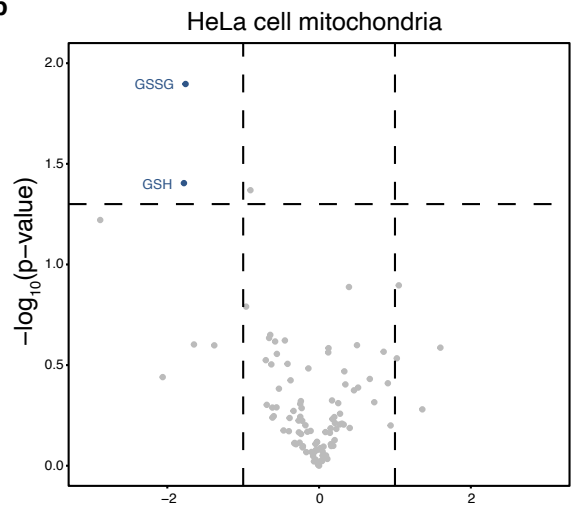

$\log _{2}$ Fold Change (SLC25A39 KO/ctrl)
GSH
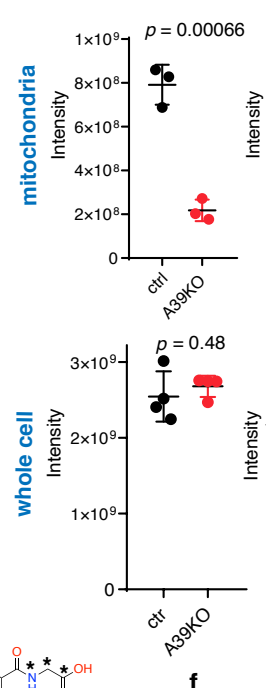

GSSG GSH/GSSG ratio

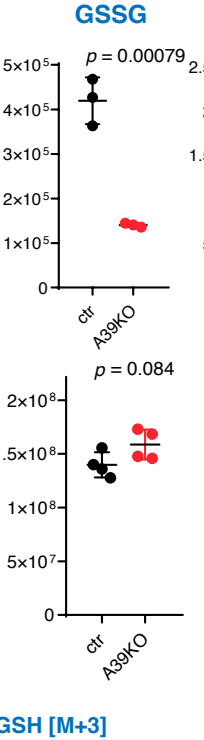

$\left.2.5 \times 10^{3}\right] \quad{ }^{p=0.29}$

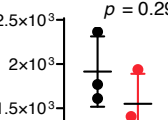

$\%$

$5 \times 10^{2}$

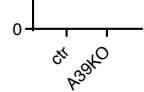

${ }^{20} 1 \begin{aligned} & p=0.37 \\ & \frac{p}{6}\end{aligned}$

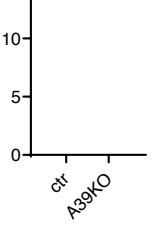

GSSG [M+3]
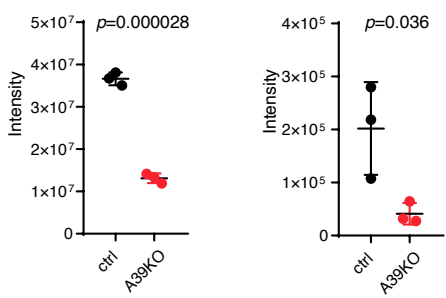

h

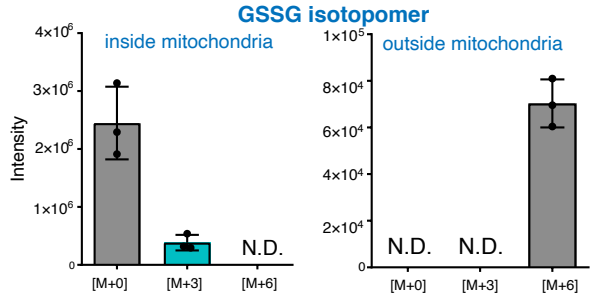


Fig. 5 | SLC25A39 is required for mitochondrial GSH transport. a and b, Volcano plots of mitochondrial metabolites from the A39 KO and control cells in K562 cells (a) and HeLa cells (b) highlighting a depletion of reduced and oxidized glutathione, GSH and GSSG respectively, in the A39 KO mitochondria. c, schematic of the biosynthesis and degradation of GSH in mammalian cells. GSH is a tripeptide $\gamma$-Glu-Cys-Gly exclusively synthesized in the cytosol by two cytosolic enzyme systems, $\gamma$-glutamylcysteine synthetase (GCLC and GCLM) and GSH synthetase (GSS) d, LC-MS measurement of reduced GSH, oxidized GSSG and GSH/GSSG ratio in isolated mitochondria and whole cells. e, Schematic overview of the organelle based GSH uptake assay. Note that the imported labeled GSH can react with endogenous, unlabeled GSH to generate GSSG. f, LC-MS measurement of mitochondrial labeled GSH and singly labeled GSSG in the uptake assay $(30 \mathrm{~min}) . \mathbf{g}$, Time-course of labeled GSH uptake into mitochondria. $\mathbf{h}$, GSSG isotopomer distribution from the inside (washed mitochondria fraction) and the outside (assay supernatant) of the control mitochondria in the GSH uptake assay at the 30 min time point. 


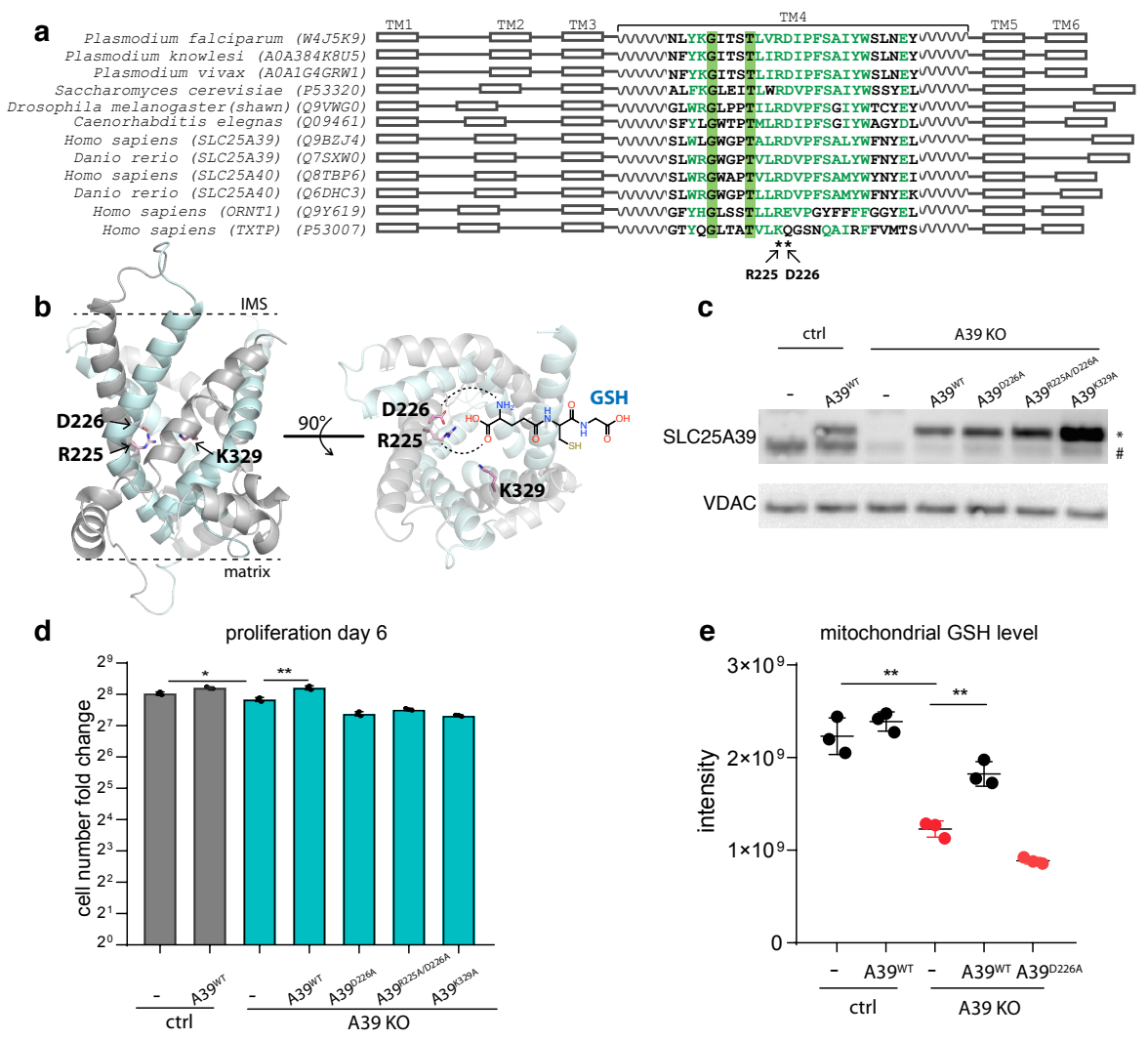

Fig. 6 | Structural modeling and functional mutagenesis of SLC25A39. a, Multiple sequence alignment of the eukaryotic A39 ortholog sequences spanning diverse taxa alongside with the amino acid ornithine transporter human SLC25A15 (ORNT1) and citrate transporter human SLC25A1 (TXTP). The positions for the human SLC25A39 Arg225 and Asp226 residues predicted to specifically bind amino acid are labeled by asterisks. b, The modeled human A39 structure based on the ANT structure c-state conformation (PDB: 1OKC), in both side view (left) and cytoplasmic/mitochondrial intermembrane space (IMS) view (right). Odd transmembrane domain (TM1, 3, 5) are shown in grey and even (TM2, 4, 6) are shown in cyan. Two predicted GSH binding residues (R225, D226) and the predicted residue critical for solute-binding induced conformational change (K329) are shown. The predicted interaction between R225 and D226 with the carboxylate and amino group of the glutamate residue of GSH is shown by dashed line c, Western blotting showing the endogenous A39 (\#) and ectopically expressed A39 protein (*). d, Rescue of growth fitness defect in the A39 KO cells by wild type A39 ${ }^{\text {WT }}$ versus A39 mutants predicted to be defective either in GSH binding (A39 ${ }^{\mathrm{D} 226 \mathrm{~A}}, \mathrm{~A} 39^{\mathrm{R} 225 \mathrm{~A} / \mathrm{D} 226 \mathrm{~A}}$ ) or in conformational changes (A39 $\left.{ }^{\mathrm{K} 329 \mathrm{~A}}\right)$. e, LC-MS measurement of mitochondrial GSH level showing that the predicted substrate binding mutant A39 ${ }^{\mathrm{D} 226 \mathrm{~A}}$ cannot restore mitochondrial GSH level in the A39 $\mathrm{KO}$ cells. 
a
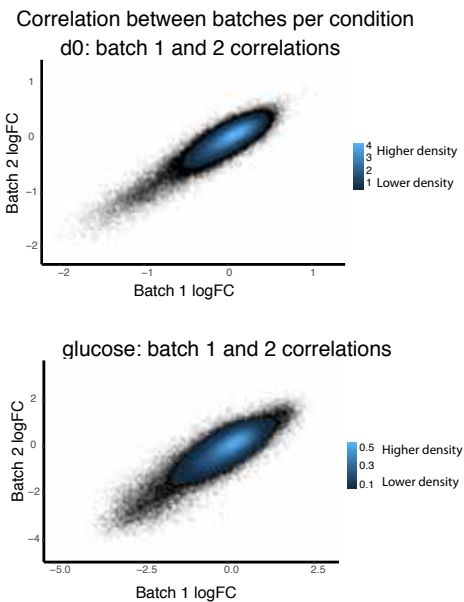

Batch $1 \operatorname{logFC}$

C Filtering procedure

$273 \times 273=74,529$ plasmids

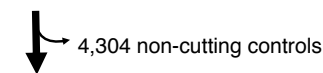
69,048

$\longrightarrow 1,016$ bad cutting controls

68,032

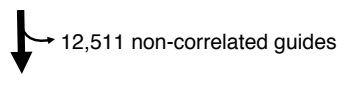

55,521

f b Correlation between Sp and Sa guides per gene
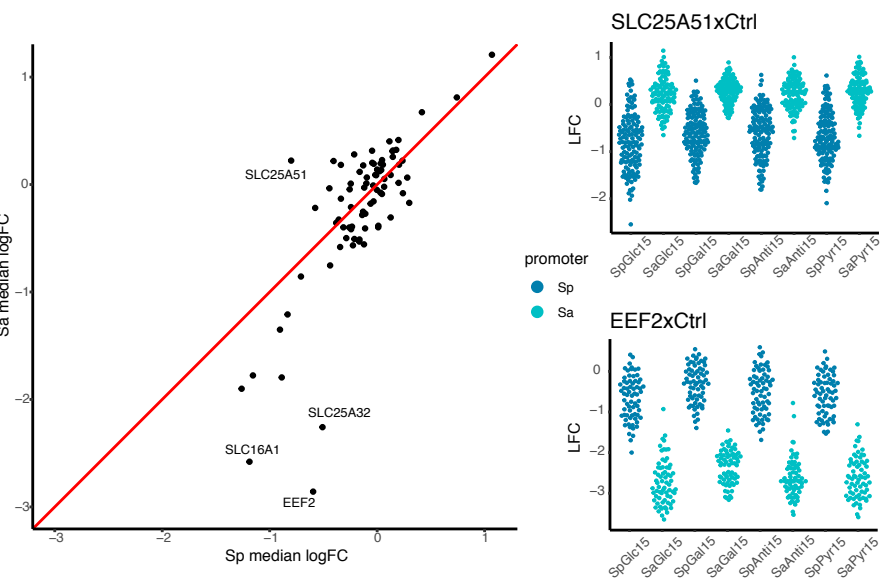

e
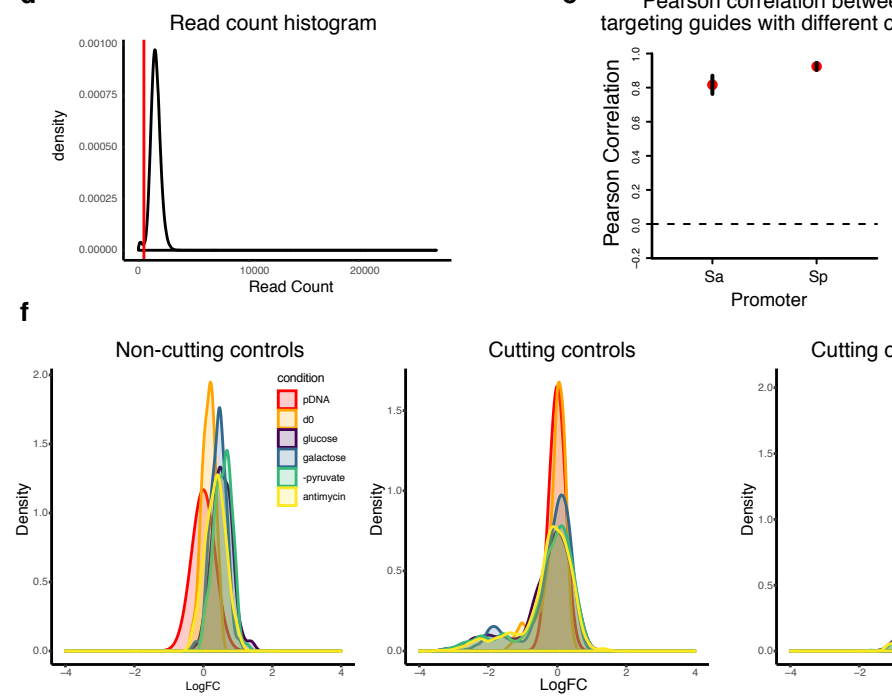

Cutting controls after filtering

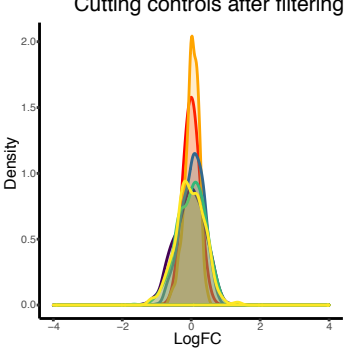

g Cutting control correlation

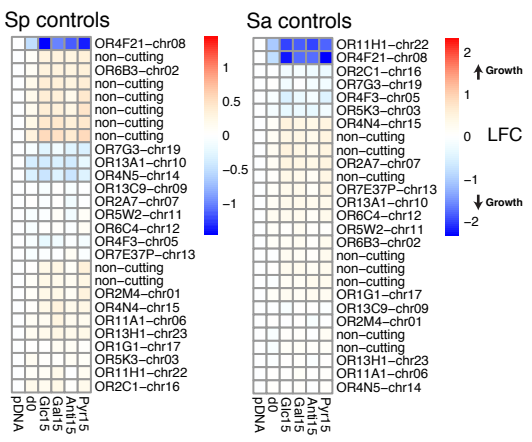

h Cutting guide correlation

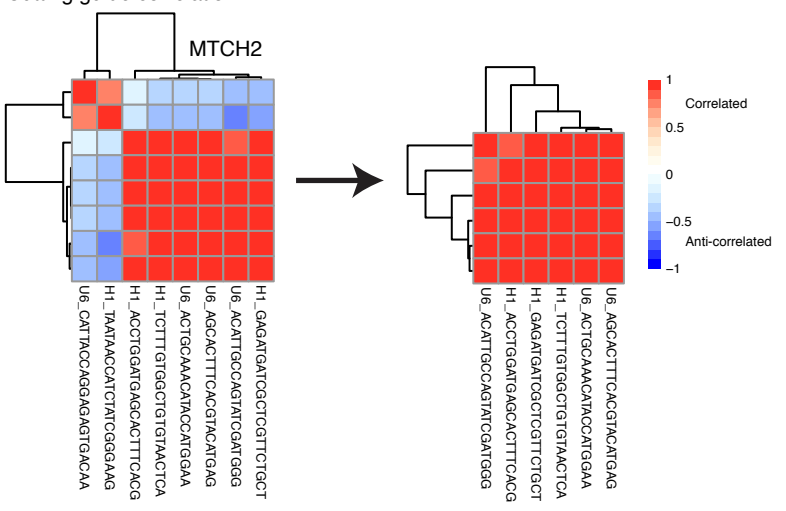

Supplementary Fig. 1 | Data analysis for the combinatorial CRISPR screen of SLC25

family. a, The correlations between the LFC values of two screen batches on day 0 and day 15 in glucose with the superimposed contour plot to show point density. Lighter blue means higher 
density of points. $\mathbf{b}$, (Left) The correlation of LFC values of the average single gene KO fitness between the Ctrl x Gene vs Gene x Ctrl. Labels indicate a very small number of genes that fall off the diagonal. (Right) For instance, SLC25A51 has a no-growth phenotype when knocked out by targeting guides at SaCas9 position, and a growth inhibition phenotype when knocked out by targeting guides at SpCas9 position. EEF2 shows a more extreme deleterious phenotype when knocked out by SaCas9 guides compared to SpCas9 guides. c, The filtering procedure for data analysis (see details in the methods). d, Read count histogram for all guide pairs in the plasmid DNA; an empirical cut-off at 500 reads is indicated with a red line. e, The Pearson correlation of the single gene KO fitness phenotype between the targeting SpCas9 guides (Gene x Ctrl) vs the SaCas9 guides (Ctrl x Gene). f, The density plots for the LFC value of non-cutting controls (left), cutting controls (middle), and cutting controls after filtering (right). Different conditions are color-coded (pDNA, red; day 0, orange; glucose, purple; galactose, blue; -pyruvate, green; antimycin, yellow). g, Heatmaps showing the LFC of the cutting negative control guides across the different conditions. Cutting control guides with deleterious fitness phenotype were filtered from both SaCas9 and SpCas9 positions. h, The Pearson correlation of SpCas9 and SaCas9 guides targeting the same single gene (either Gene x Ctrl or Ctrl x Gene) across different conditions were calculated. Guides that showed anti-correlation were filtered out for subsequent analysis. 
a

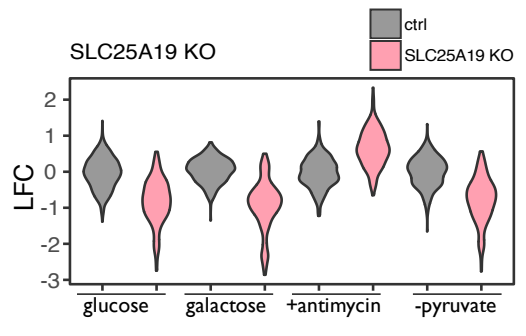

c

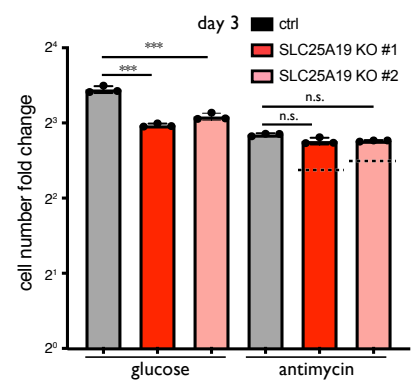

e

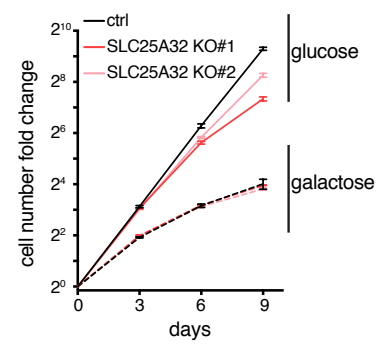

g long-term galactose adaptation

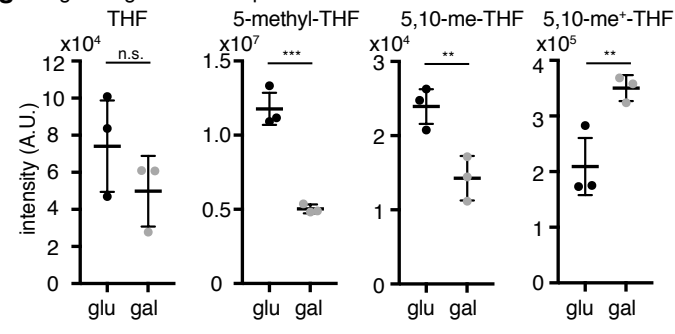

b

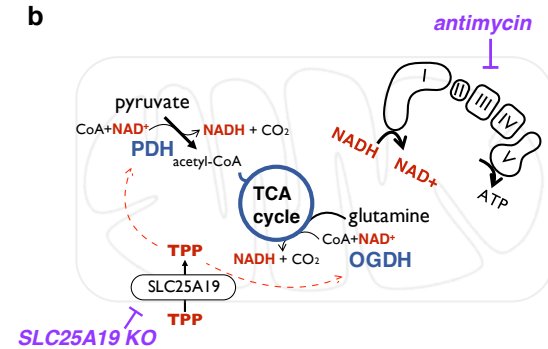

d
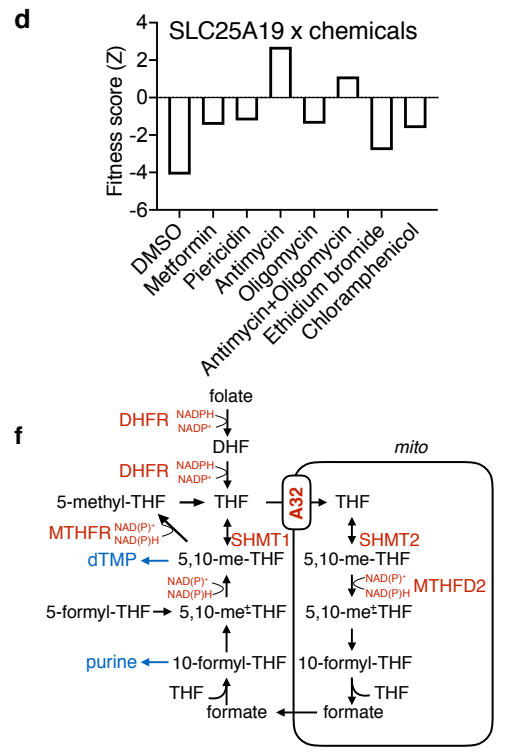

h long-term galactose adaptation

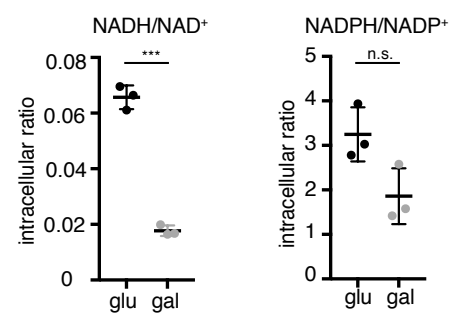

i MS/MS comparison for folate species
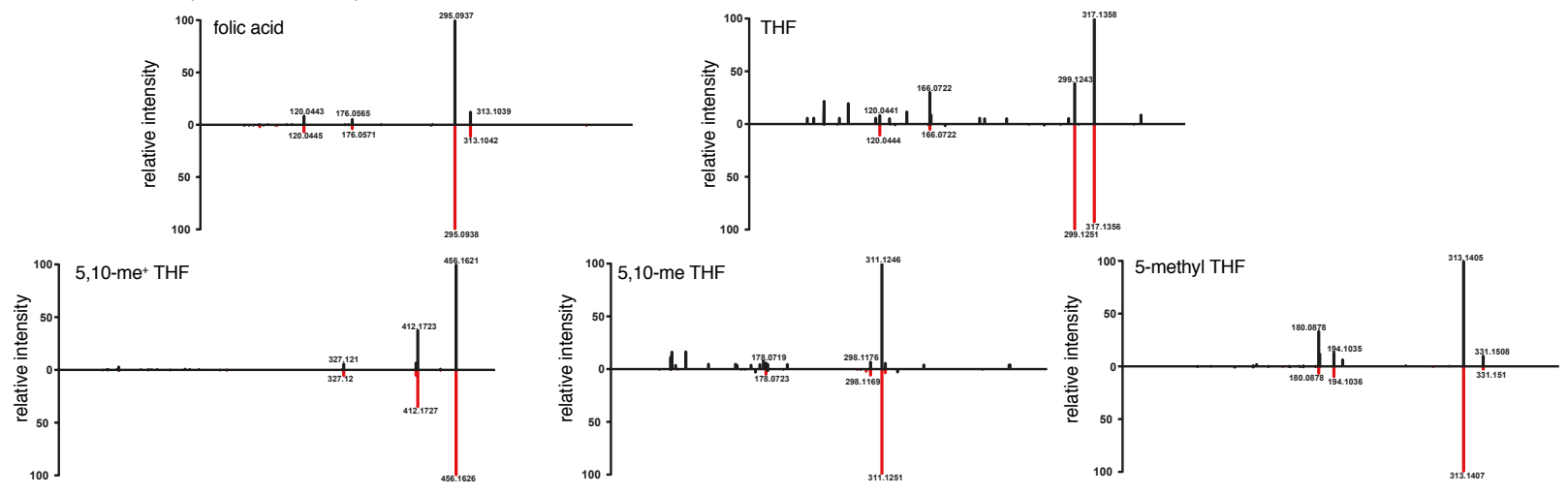

Supplementary Fig. 2 | Single KO gene by environment interaction for the two hits, SLC25A19 and SLC25A32. a, The growth fitness of SLC25A19 single CRISPR KO cells and controls in the screen. Grey is the Log Fold Change (LFC) of the Ctrl x Ctrl cells, and pink is 
LFC of the SLC25A19 KO (SLC25A19 x Ctrl or Ctrl x SLC25A19). b, Diagram depicting mitochondrial ThPP metabolism. SLC25A19 is the mitochondrial carrier for ThPP, the cofactor critical for matrix PDH and OGDH complexes that generate reduced NADH for the ETC. c, Follow-up study confirms the growth defect of SLC25A19 CRISPR KO cells using two different sgRNA guides, and the defect is buffered in the antimycin condition. d, reanalysis of the ETC inhibitor-dependent genome-wide CRISPR screen. e, The growth curve of SLC25A32 KO and control cells in glucose and galactose. $\mathbf{f}$, cellular folate metabolic pathway. $\mathbf{g}$, Long-term adaptation in galactose for 2 weeks reduced most tetrahydrofolate species, with the exception of 5,10-me ${ }^{+}$-THF. h, Long-term adaptation in galactose for 2 weeks reduced the cellular $\mathrm{NADH} / \mathrm{NAD}^{+}$and NADPH/NADP ${ }^{+}$ratio. i, MS/MS comparison of folate species detected in the samples (black) and standards (red). 


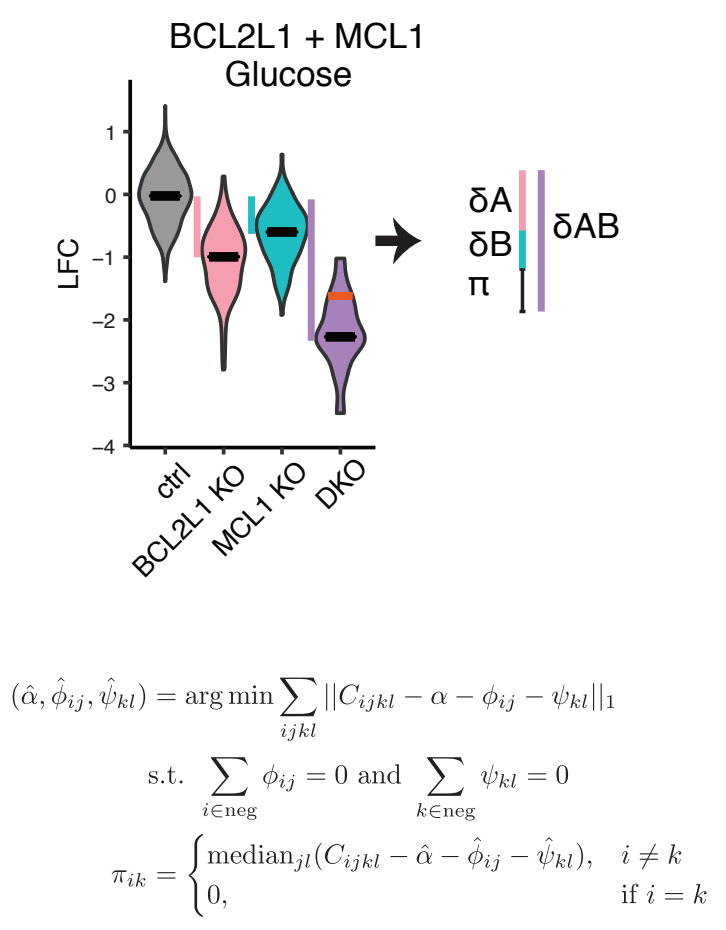

Supplementary Fig. 3 | The $\pi$-score as a measure of genetic interaction as reported in Fischer et al. 2015. The $\pi$-score is a robust estimation of the difference between the addition of two single knockout phenotypes and the observed double knockout phenotype. The $\pi$-score is calculated using the above robust linear model, where, for gene $i$ with guide $j$, and gene $k$ with guide 1 , the log fold change of the double knock outs $\left(\mathrm{C}_{\mathrm{ijk} \mathrm{l}}\right)$ are the data in the matrix. $\alpha$ is an intercept term, and $\varphi$ and $\psi$ represent the single gene terms. The $\pi$-score for a double knock-out of gene $\mathrm{i}$ with gene $\mathrm{k}$ is the median over all guides $\mathrm{j}$ and $\mathrm{l}$ of the difference between the $\log$ fold change and the estimated parameters. $\pi$-scores are defined as 0 for auto-double-knockouts. The positive control pair BCL2L and MCL1 was used to illustrate the calculation. 
a

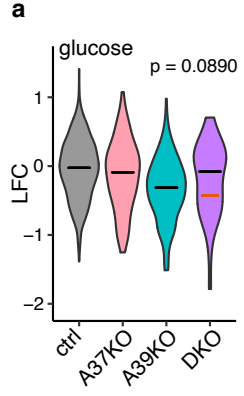

d

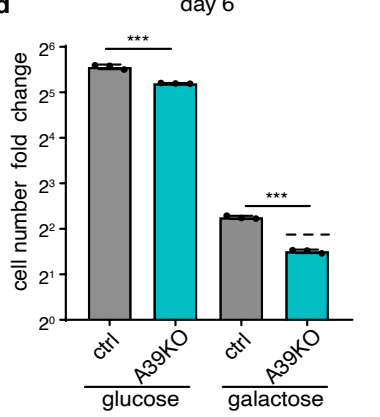

f

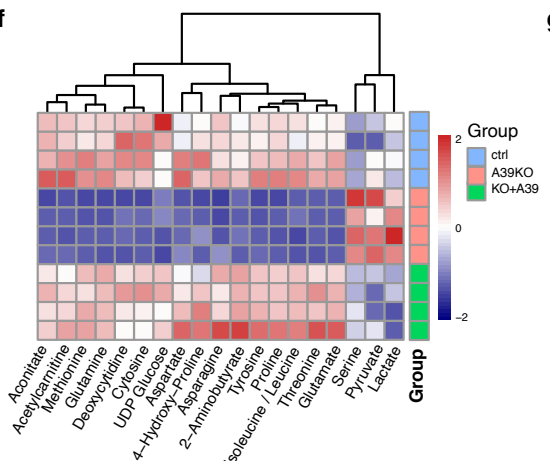

SLC25A37 + SLC25A39

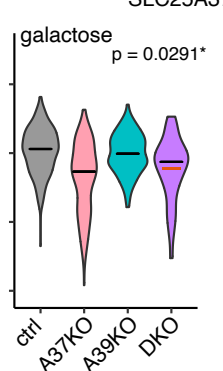

e

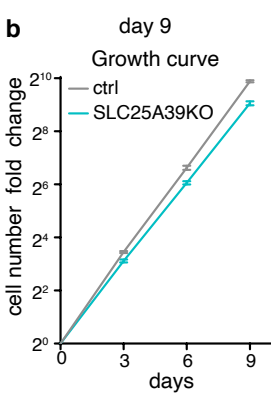

OCR (FCCP)

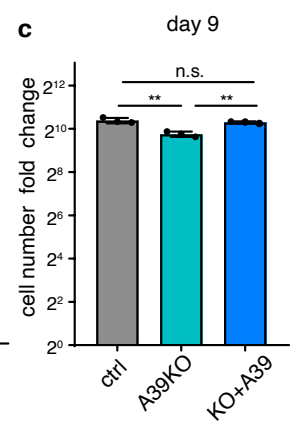

ECAR (Baseline)
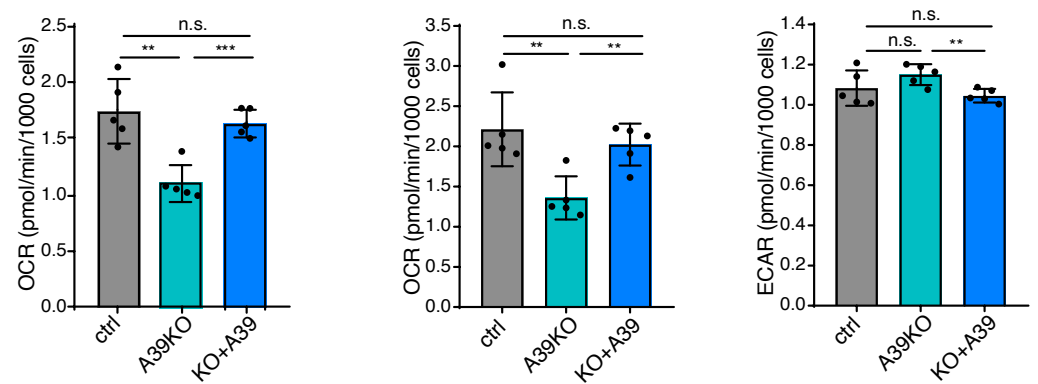

g mitochondrial isolation by mitolP $h$

Hela cell mitochondria
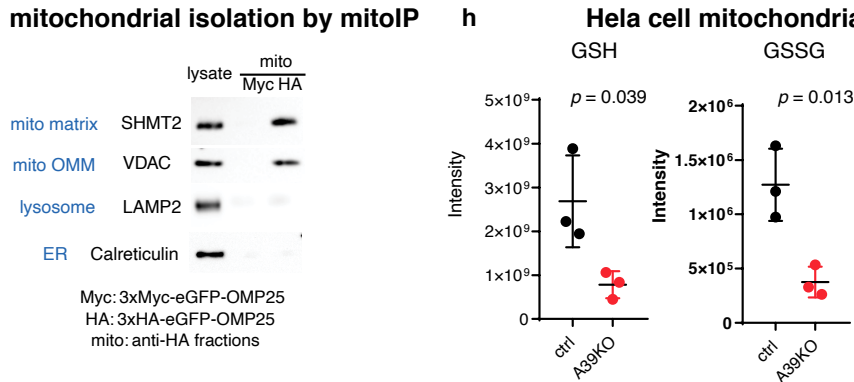

i

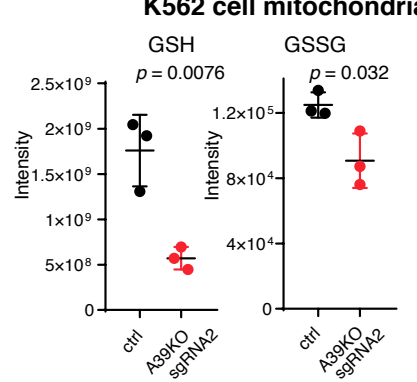

GSSG isotopomer

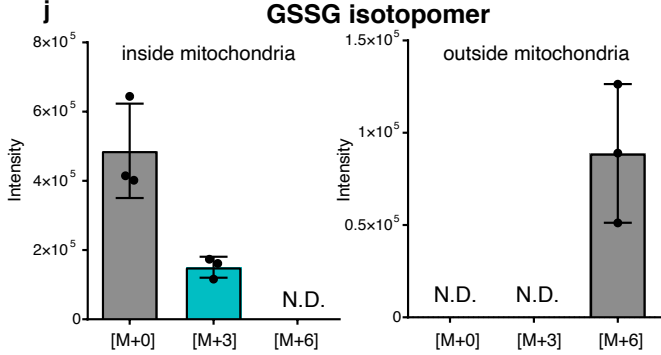

$\mathbf{k}$

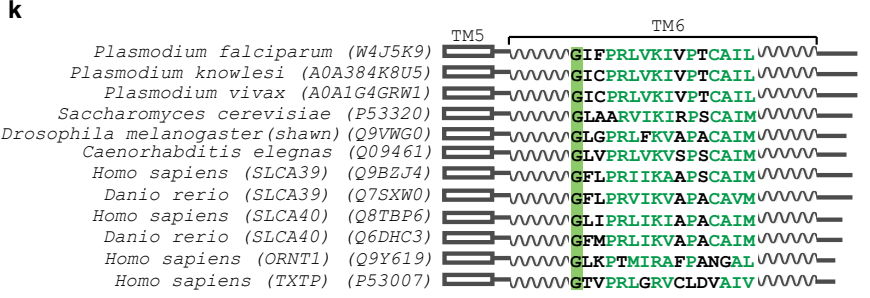

К ${ }^{*} 29$ 


\section{Supplementary Fig 4 | Validation of interactions involving SLC25A39 and functional} follow-up. a, SLC25A39 exhibits buffering interaction with SLC25A37 in the CRISPR screen, with a strongest interaction observed in the antimycin condition. b, Growth fitness of SLC25A39 CRISPR KO cells cultured in glucose condition. c, Growth fitness defect in the SLC25A39 CRISPR KO cells is rescued by re-expressing CRISPR-resistant SLC25A39. d, SLC25A39 KO cells exhibited stronger growth fitness defect in galactose condition, supporting its role in OXPHOS. e, Respiration phenotypes (Basal OCR, maximal respiration and basal ECAR) in the SLC25A39 KO are rescued by re-expressing SLC25A39. f, Heatmap showing changes in cellular metabolites caused by SLC25A39 KO and restored by re-expressing SLC25A39. g, Western blotting of organelle markers, SHMT2 (matrix), VDAC (outer-mitochondrial membrane, OMM), LAMP2 (lysosome) and Calreticulin (endoplasmic reticulum, ER), from the whole cell lysates and anti-HA immuno-isolated fractions from the cells expressing $3 \times$ MyceGFP-OMP25 (Myc) and 3xHA-eGFP-OMP25 (HA), confirming selective enrichment for the mitochondria. h, LC-MS measurement of mitochondrial GSH and GSSG levels the control and A39 KO HeLa cell mitochondria. i, LC-MS measurement of mitochondrial GSH and GSSG levels in the control and A39 KO K562 cells, in which a different A39-targeting sgRNA guide was used. j, GSSG isotopomer distribution from the inside (washed mitochondria fraction) and the outside (assay supernatant) of the A39 KO mitochondria in the GSH uptake assay at the 30 min time point. $\mathbf{k}$, Multiple sequence alignment of the eukaryotic A39 ortholog sequences spanning diverse taxa, as well as the human amino acid ornithine transporter SLC25A15 (ORNT1) and the human citrate transporter SLC25A1 (TXTP). The position for the human SLC25A39 Lys329, a positive residue previously shown to be responsible for mediating soluteinduced conformational changes and critical for transport activity is labeled with an asterisk. 
Supplementary Fig. 5 - 8 | Genetic interaction for all the gene-pairs in each media condition. Heatmaps with the $\pi$-score for each calculated genetic interaction are shown, separated by condition. The single-knockout phenotype of Ctrl x Gene or Gene $\mathrm{x} C \mathrm{trl}$ is annotated on the left side; the top dendrogram indicates the clustering of the SLC25 family members by sequence homology. The diagonal is greyed out.

Supplementary Table 1 | Raw data from combinatorial CRISPR screen

Supplementary Table 2 | Summary of all single KO gene $x$ environment interactions

Supplementary Table 3 | $\pi$-score and p-values for all gene $\mathbf{x}$ gene interactions within each condition

Supplementary Table 4 | Summary of all gene $x$ gene $x$ environment interactions

Supplementary Table 5 | Expression profiles of SLC25 genes in K562 cells 


\section{Supplementary Notes}

\section{Other observations about the GxGxE screen}

None of the 12 non-expressed SLC25 genes (Supplementary Table 5) were recovered as hits in any of the conditions, supporting the robustness of our CRISPR screen and data analysis pipeline. Our GxE and GxGxE screen hits did not include SLC25 genes that we initially anticipated. For instance, for SLC25A51, a recently identified carrier for NAD cofactor ${ }^{56-58}$, our screen confirmed a fitness defect of SLC25A51 KO using the spCAS9 guides (Supplementary Fig. 1b), but did not reveal a GxE interaction in galactose, probably due to redundant mechanisms supporting NAD transport as well as a mild OXPHOS defect of the SLC25A51 KOs in the screen cell line. We did not observe genetic interaction between SLC25A12 and SLC25A13, two Glutamate/Aspartate exchangers in the malate-aspartate shuttle, perhaps because of the functional redundancy with other glutamate carriers SLC25A18 and SLC25A22 ${ }^{59}$ and the aspartate carrier ${ }^{60}$. Our hits did not include another recently identified branched-chain amino acid (BCAA) carrier SLC25A44 ${ }^{61}$, probably because BCAA metabolism is dispensable for the K562 cell fitness. In our single gene KO analysis, while the loss of SLC25A25 (ATP-Mg/Pi carrier), SLC25A33 and SLC25A36 (two pyrimidine carriers) conferred a mild growth advantage in the screen (Fig. 2a), we were not able to validate the growth benefit effects of SLC25A33 and SLC25A36 single KOs in the follow-up experiments, presumably due to a "paracrine"-like effect from other genotypes in the pooled screening format. It is unknown if the growth benefit in the SLC25A33 KO in the screen might be linked to a recent proposed role of SLC25A33 in regulating pyrimidine imbalance and innate immunity $^{62}$. In our screen, we did not observe a genetic interaction between SLC25A39 and its paralogous gene SLC25A40. 


\section{Methods}

Cell culture K562 cells (CCL-243) and HEK293T cells were obtained from the ATCC and maintained in DMEM (Thermo Fisher, 11995) + 10\% FBS (Sigma, F2442) + 100 U/ml Pen/Strep (Thermo Fisher, 15140122). The cells were tested for mycoplasma periodically. The four media conditions used in the screen and in the follow-up experiments were prepared with following reagents: DMEM (Thermo Fisher, 11966), glucose (Thermo Fisher, A2494001), galactose (Teknova, G0505), dialyzed FBS (dFBS, Thermo Fisher, 26400044), sodium pyruvate (Thermo Fisher, 11360070), and uridine (Sigma, U3750). The four media conditions are:

(1) Glc: $25 \mathrm{mM}$ glucose, $10 \% \mathrm{dFBS}, 1 \mathrm{mM}$ sodium pyruvate, $50 \mathrm{ug} / \mathrm{ml}$ uridine, and $100 \mathrm{U} / \mathrm{ml}$ Pen/Strep

(2) Gal: $25 \mathrm{mM}$ galactose, $10 \% \mathrm{dFBS}, 1 \mathrm{mM}$ sodium pyruvate, $50 \mathrm{ug} / \mathrm{ml}$ uridine, and $100 \mathrm{U} / \mathrm{ml}$ Pen/Strep

(3) Antimycin: $25 \mathrm{mM}$ glucose, 10\% dFBS, $1 \mathrm{mM}$ sodium pyruvate, $50 \mathrm{ug} / \mathrm{ml}$ uridine, and 100 $\mathrm{U} / \mathrm{ml}$ Pen/Strep with $100 \mathrm{nM}$ antimycin (Sigma, A8674)

(4) -Pyruvate: $25 \mathrm{mM}$ glucose, 10\% dFBS, $50 \mathrm{ug} / \mathrm{ml}$ uridine, and $100 \mathrm{U} / \mathrm{ml}$ Pen/Strep

For folate experiment, RPMI1640 media lacking glutamine, glucose, vitamin $\mathrm{B}_{12}$, folic acid, phenol red (US Biological, R9001-02) were further supplemented with $300 \mathrm{mg} / \mathrm{l}$ glutamine (Thermo Fisher, 25030081), $25 \mathrm{mM}$ glucose, $0.005 \mathrm{mg} / 1$ vitamin $\mathrm{B}_{12}$ (Sigma, V6629), 10\% dFBS, $50 \mathrm{ug} / \mathrm{ml}$ uridine, $100 \mathrm{U} / \mathrm{ml} \mathrm{Pen} /$ Strep and either $10 \mu \mathrm{M}$ (high folate media) or $0.08 \mu \mathrm{M}$ (low folate media) folic acid (Sigma, F8758). Methotrexate (Sigma, A6770) was used in high folate media to inhibit the folate pathway. 5-aminolevulinic acid (ALA, Sigma, A7793) was used to upregulate the heme biosynthesis pathway.

For SLC25A39 characterization, both RPMI (Gibco, 11875) -based and DMEM (Gibco, 11995)based media are used interchangeably with little difference, except for Fig. 4g and Supplementary Fig. 4f were shown for the RPMI condition. For K562 erythroid differentiation experiment, K562 cells were treated with $0.5 \mathrm{mM}$ sodium butyrate (Sigma, 303410), $0.5 \mathrm{mM}$ 5-aminolevulinic acid (Sigma, A7793), or the combination of both for three days before the assay. For K562 iron toxicity experiment, K562 cells were treated with $40 \mathrm{mg} / \mathrm{L}$ ammonium iron(III) citrate (Sigma, F5879), which is approximately equivalent to $125 \mu \mathrm{M}$ ferric salt.

Lentivirus for the follow-up experiments was produced using HEK 293T cells. Specifically, $1 \mathrm{x}$ $10^{6}$ HEK293T cells were plated in $6 \mathrm{~cm}$ dish one day before induction. A mixture with $1 \mu \mathrm{g}$ VSV$\mathrm{G}$ (Addgene, 8454), $1 \mu \mathrm{g}$ psPAX2 (Addgene, 12260), $2 \mu \mathrm{g}$ lentiviral plasmids and $10 \mu \mathrm{l} \mathrm{dH}_{2} \mathrm{O}$ was further added with $150 \mu \mathrm{l}$ serum free DMEM and $9 \mu \mathrm{l}$ X-tremeGENE HP DNA Transfection Reagent (Sigma, 6366244001), and incubated for 15-30 min at room temperature before adding drop-wise to the cells. Two days after induction, the spent media was collected and filtered through $0.45 \mu \mathrm{m}$ sterile filter, and the resulting virus can be stored at $-80{ }^{\circ} \mathrm{C}$ till use.

Combinatorial CRISPR screen vectors The vector used for combinatorial CRISPR screens has been reported before ${ }^{1}$ and is available through Addgene: pPapi (also known as pXPR_207). In this vector, U6 and H1 promoters express two sgRNAs; short EF1a promoter (EFS) expresses SaCas92A-PuromycinR (Addgene, 96921). 
pPapi-SLC25 library construction and production To construct the pPapi-SLC25 library, SpCas9 and SaCas9 guides were designed using the GPP sgRNA designer (https://broad.io/gppsgrna-design) with a quota of four guides per gene per enzyme. 53 SLC25 family members and 7 additional SLC proteins present in the Mitocarta 2.02 were included, as well as two guides per gene for BCL2L1, MCL1 and EEF2 as positive controls; 8 different non-cutting controls and 19 cutting controls that target olfactory receptors (ORs) across different chromosomes served as negative controls.

Pooled libraries for expression of single sgRNAs were made as previously described ${ }^{1}$, with oligonucleotide pools obtained from CustomArray16. For cloning of Big Papi pools, oligonucleotide inserts (Ultramers, IDT) were designed with 5' BsmBI sites followed by 20 or 21 nt crRNA, $82 \mathrm{nt}$ tracrRNA, $6 \mathrm{nt}$ barcode, and a $17 \mathrm{nt}$ complementary sequence (see Supplementary Note 1 in Najm et al. 2018 paper). The oligonucleotides for SpCas9 sgRNAs and SaCas9 sgRNAs were separately mixed together at a concentration of $5 \mu \mathrm{M}$ each. $10 \mu \mathrm{l}$ of each pool of oligonucleotides was then combined in a $100 \mu$ reaction and extended using NEBNext (New England Biolabs) with an annealing temperature of $48^{\circ} \mathrm{C}$. The resulting dsDNA was purified by spin-column then ligated into the BsmBI-digested pPapi vector using 100 cycles of Golden Gate assembly with $100 \mathrm{ng}$ insert and $500 \mathrm{ng}$ vector using Esp3I and T7 ligase. The DNA was isopropanol precipitated and electroporated into STBL4 cells. A zero-generation (G0) plasmid DNA pool was then amplified by a second electroporation into STBL4 cells to create the G1 plasmid DNA pool, which was then used for virus production.

Screen virus production For individual virus production: $24 \mathrm{~h}$ before transfection, HEK293T cells were seeded in 6-well dishes at a density of $1.5 \times 10^{6}$ cells per well in $2 \mathrm{ml}$ of DMEM $+10 \%$ FBS. Transfection was performed using TransITLT1 (Mirus) transfection reagent according to the manufacturer's protocol. In brief, one solution of Opti-MEM (Corning, $66.25 \mu \mathrm{l})$ and LT1 (8.75 $\mu \mathrm{l}$ ) was combined with a DNA mixture of the packaging plasmid pCMV_VSVG (Addgene, 8454, $250 \mathrm{ng}$ ), psPAX2 (Addgene, 12260, 1,250 ng), and the sgRNA containing vector (e.g., pPapi, $1,250 \mathrm{ng}$ ). The two solutions were incubated at room temperature for 20-30 min, during which time the HEK293T cells were replenished with fresh media. After this incubation, the transfection mixture was added dropwise to the surface of the HEK293T cells, and the plates were centrifuged at $1,000 \mathrm{~g}$ for $30 \mathrm{~min}$. Following centrifugation, plates were transferred to a $37^{\circ} \mathrm{C}$ incubator for $6-$ $8 \mathrm{~h}$, then the media was removed and replaced with media supplemented with 1\% BSA. A largerscale procedure was used for production of the sgRNA library; $24 \mathrm{~h}$ before transfection, $18 \times 10^{6}$ HEK293T cells were seeded in a $175 \mathrm{~cm}^{2}$ tissue culture flask, with transfection performed as described above using $6 \mathrm{ml}$ of Opti-MEM and $300 \mu$ of LT1. Flasks were transferred to a $37{ }^{\circ} \mathrm{C}$ incubator for 6-8 h, then the media was aspirated and replaced with BSA-supplemented media. Virus was harvested $36 \mathrm{~h}$ after this media change.

Genomic DNA (gDNA) preparation At least $7.5 \times 10^{7}$ cells from the screen were washed with PBS, and the gDNA was first isolated using Blood \& cell culture DNA maxi kit (Qiagen, 13362) as per the manufacturer's instructions. The gDNA precipitant was suspended in $600 \mu \mathrm{H}_{2} \mathrm{O}$ overnight, and subject to chloroform purification. Specifically, the suspended DNA was mixed with $600 \mu \mathrm{l}$ phenol:chloroform:isoamyl alcohol (25:24:1) (Sigma, P2069-100ml) in the fume hood by inverting the tube. The tube was further incubated at room temperature for $5 \mathrm{~min}$, and spun at highest speed at room temperature using table top centrifuge for $15 \mathrm{~min}$. The top aqueous phase 
$(\sim 540 \mu \mathrm{l})$ was collected, mixed with $60 \mu \mathrm{l} \mathrm{NaAcetate}(3 \mathrm{M} \mathrm{pH} 5.5), 2 \mu \mathrm{l}$ glycogen $(20 \mu \mathrm{g} / \mu \mathrm{l})$ and $600 \mu \mathrm{l}$ isopropanol, before frozen at $-80{ }^{\circ} \mathrm{C}$ for at least one hour. After thawing, the DNA pellet was washed with $1 \mathrm{ml}$ cold $70 \%$ ethanol, air-dried, and suspended in $600 \mu$ ultrapure $\mathrm{H} 2 \mathrm{O}$ overnight. The gDNA was quantified using Qubit dsDNA HS Assay Kit (Thermo Fischer).

PCR of the sgRNA regions, NGS and deconvolution For the pPapi vector, dual sgRNA cassettes and plasmid DNA (pDNA) were PCR-amplified and barcoded with sequencing adaptors using ExTaq DNA Polymerase (Clontech) following the same procedure as Supplementary Note 1 in Najm et al. 2018 paper except the sequencing primer. The primer sequence used here uses a shorter read length and includes the super $\mathrm{T}$ modified bases to stabilize the duplex: CAACTTGAAAAAGTGGCACCGAGTCGGTGC T/iSuper-dT/TT/iSuper-dT/T. This primer binds immediately upstream of the $6 \mathrm{nt}$ barcode for the SpCas 9 guide, then reads the constant overlap extension region, and finally the $6 \mathrm{nt}$ barcode for the SaCas9 guide.

Specifically, amplified samples were then purified with Agencourt AMPure XP SPRI beads (Beckman Coulter, A63880) according to manufacturer's instructions and sequenced on a NextSeq sequencer (Illumina) with 50 nt single-end reads, with a $10 \%$ spike-in of PhiX DNA. Deconvolution of single sgRNA expressing vectors was as described before ${ }^{1}$. For the pPapi vector, reads of the first sgRNA were counted by first searching in the sequencing read for CACCG, the part of the vector sequence that immediately precedes the 20-nucleotide U6 promoter-driven SpCas9 sgRNA. The sgRNA sequence following this search string was mapped to a reference file with all sgRNAs in the library. To find the H1 promoter-driven SaCas9 sgRNA, two 21-nucleotide sequences were compared: the sequence beginning 194 nucleotides after the SpCas9 sgRNA and the sequence following the $\mathrm{S}$. aureus tracr sequence (CTTAAAC). If the sequences matched, the $21 \mathrm{nt}$ sequence was then mapped to the reference file with all SaCas9 sgRNA. For some sequencing lanes with poorer quality, the reference file with the SaCas9 sgRNAs sequences was shortened, such that fewer than $21 \mathrm{nts}$ were needed to match in order to determine the identity of the sgRNA in that position. Reads were then assigned to the appropriate experimental condition based on the 8-nucleotide P7-appended barcode.

CRISPR screen Two independent replicates were performed. For each replicate, K562 cells expressing Cas9-2A-EGFP ${ }^{3}$ were first sorted for GFP-expressing cells, and within one week after sorting, the cells were spin-infected with the pPapi-SLC25 CRISPR lentiviral library. Specifically, approximately 2-3 x $10^{8}$ cells were aliquoted in 12 well plates with $3 \times 10^{6}$ cells in each well for up to 96 wells, and further added with virus volume corresponding to $20-30 \%$ infection efficiency and polybrene at a final concentration at $5 \mu \mathrm{g} / \mathrm{ml}$. Together, each well holds $2 \mathrm{ml}$ infection mixture, and all the 12 well plates were spun at $1000 \mathrm{~g}$ for $2 \mathrm{hr}$ at $37^{\circ} \mathrm{C}$. After the spin, the media were carefully removed and replaced with $2 \mathrm{ml}$ fresh media, followed by incubation overnight. The infected cells were selected for 4 days with $2 \mathrm{ug} / \mathrm{ml}$ puromycin (Thermo Fischer, A1113803) at a seeding density of $1 \times 10^{5}$ per $\mathrm{ml}$ in T175 flask, and further expanded in regular cell culture media for 3 days at a seeding density of $1 \times 10^{5}$ per $\mathrm{ml}$, and this time point is considered $\mathrm{d} 0$. Then for the growth fitness screen, the cells were washed with PBS, and cultured for additional 15 days ( $\mathrm{d}$ 15) into four different media conditions mentioned above in spinner flask (Corning, CLS3561) with vent caps (Corning, CLS3567). During the screen, in order to keep the cells at the exponential growth phase, cells were passaged every three days and seeded at following density: $1 \times 10^{5}$ per $\mathrm{ml}$ for glucose, $2 \times 10^{5}$ per $\mathrm{ml}$ for galactose, $1.5 \times 10^{5}$ per $\mathrm{ml}$ for antimycin, and $1 \mathrm{x}$ 
$10^{5}$ per $\mathrm{ml}$ for -pyruvate. During each passage, $\sim 10 \%$ of the spent media were kept and mixed with the fresh media. For each time point, approximately $7.5 \times 10^{7}$ cells were pelleted at $300 \mathrm{~g}$ for $10 \mathrm{~min}$ and washed with $10 \mathrm{ml}$ PBS for subsequent genomic DNA preparation.

Screen data analysis and QC To infer the relative growth fitness phenotypes, we quantified and $\log 10$-normalized sgRNA plasmid abundance at day 15 in respective media conditions, and compared to the starting abundance (plasmid DNA). The raw data consisted of the read counts of 273 guides built into dual-guide plasmids, giving $273 \times 273=74,529$ total guide-guide pairs. The log-fold-change of the raw data was calculated:
Log fold change (LFC) $\log 2($ frequency_pDNA $\times 1000000+1)$
$\log 2$ (frequency_condition $\times 1000000+1) \quad-$

The following filtering steps were performed to decrease noise and increase power of detection (see Supplemental Fig. 1c):

(1) Guide-guide pairs with a low read count in the pDNA were filtered using an empirical cutoff based on the read count distribution. Individual guides with median read counts lower than the empirical cut-off were also filtered $(1,177)$.

(2) Plasmids which contained a non-cutting control guide were filtered $(4,304)$.

(3) A small number of cutting control guides showed lethal single-gene phenotypes, and were subsequently filtered $(1,016)$

(4) For each gene in each batch, across all conditions, a correlation matrix between all guide sequences was calculated. When the sum of the correlations for a guide sequence across a row was less than 0 , the guide-guide pair was marked as "poor" since the guide-guide pair did not correlate with itself over multiple conditions. All unique guides marked as poor in either batch $1(\mathrm{n}=40)$ or batch $2(\mathrm{n}=39)$ were removed (unique $\mathrm{n}=50)$. In total, 12,511 non-correlated guide-guide pairs were removed in this step.

The remaining 55,521 guide-guide pairs were used in subsequent analysis. It was confirmed that no gene or gene pair was unrepresented in the data. For all downstream analyses, GeneA x GeneB pair and GeneB x GeneA pair are combined.

It is worth noting that the presence of both cutting negative control sgRNA guides and non-cutting negative control sgRNA guides in our library allowed experimental comparison between the two types of negative controls. Previous genome-wide CRISPR screens suggest that double-stranded DNA cutting by Cas9 enzymes might lead to deleterious effects on cell proliferation ${ }^{4}$, and thus control guides that cut the genome might be better negative controls than non-cutting control guides. Indeed, in our screen, the 19 cutting controls that target olfactory receptors (ORs) across different chromosomes showed less variation within and across conditions and also confer a growth phenotype very close to neutral $(\mathrm{LFC}=0)$. On the other hand, the cells bearing the noncutting control guides appear to grow better than the population mean over time (Supplementary Fig. 1f). For this reason, we only used cutting guides for downstream analysis, and removed any control guides that showed deleterious phenotypes.

After the quality control and filtering steps mentioned above, the two batches of the screen were found to correlate highly (Pearson's R=0.86, two-tailed p-value $<2.2 \mathrm{e}-16$, Supplementary Fig. 1a). 
For each gene, there is high correlation between the SaCas9 and SpCas9 guides, as the median LFC of the Gene x Ctrl plasmids were well-correlated to the Ctrl x Gene plasmids (Pearson's $\mathrm{R}=0.78$, two-tailed p-value $<2.2 \mathrm{e}-16$, Supplementary Fig. $1 \mathrm{~b}$ ). Within each Cas9 position, the sgRNA guides were more correlated in the SpCas9 position (mean Pearson's $\mathrm{R}=0.92$ ) than in the SaCas9 position (mean Pearson's $\mathrm{R}=0.82$; two-tailed t-test p-value $<2.2 \mathrm{e}-16$, Supplementary Fig. 1e).

GxE interactions data analysis In order to assay for gene-by-environment interaction, we utilized a subset of our dataset which included only guide-guide pairs where at least one guide was a control guide, giving us single-knock-outs and the ctrl-ctrl pairs. Since growth rates differed between conditions, we took the z-score of the LFC within a condition to compare across conditions. The z-score was calculated relative to the control-control distribution in each condition, which was thought to best reflect the neutral growth phenotype within a condition. We then performed the following regression:

$$
\text { z-score } \sim \text { gene }+ \text { gene:condition }
$$

GxG interactions data analysis Gene-gene interactions were modelled using a previouslydescribed scoring system called the pi-score ${ }^{5}$ :

$$
\begin{aligned}
&\left(\hat{\alpha}, \hat{\phi}_{i j}, \hat{\psi}_{k l}\right)= \arg \min \sum_{i j k l}\left\|C_{i j k l}-\alpha-\phi_{i j}-\psi_{k l}\right\|_{1} \\
& \text { s.t. } \sum_{i \in \mathrm{neg}} \phi_{i j}=0 \text { and } \sum_{k \in \text { neg }} \psi_{k l}=0 \\
& \pi_{i k}= \begin{cases}\operatorname{median}_{j l}\left(C_{i j k l}-\hat{\alpha}-\hat{\phi}_{i j}-\hat{\psi}_{k l}\right), & i \neq k \\
0, & \text { if } i=k\end{cases}
\end{aligned}
$$

Where, for gene $i$ with guide $j$, and gene $k$ with guide $l$, the log fold change of the double knock outs $\left(\mathrm{C}_{\mathrm{ijk} \mathrm{k}}\right)$ are the data in the matrix. Alpha is an intercept term, and phi and psi represent the single gene terms. Robust linear regression is used to estimate alpha, phi, and psi across the dataset, subject to the conditions that control genes have a distribution centered at 0 . The pi-score for a double knock-out of gene $i$ with gene $k$ is the median over all guides $j$ and $l$ of the difference between the log fold change and the estimated parameters. Pi-scores are not defined as 0 for autodouble-knockouts. Significance is estimated from the guide-level data using the limma package to create a linear model within R. Significance values from the regression were then corrected for false discovery rate using the Benjamini-Hochberg method (the default within limma); it is these adjusted $p$-values which are reported. The effect size is also reported. All data analysis was done using custom $\mathrm{R}$ scripts performing standard analyses using $\mathrm{R}$ version 3.6.0 and Bioconductor release 3.9.

GxGxE interaction filtering Gene-gene interaction can also vary by condition. In order to look at gene-gene interaction in the context of different environments, we first restricted ourselves to all gene-gene interactions with FDR $<2 \%$ that included only SLC25 family member genes. Then we removed gene-gene interactions that met the following disqualification criteria:

(1) Small effect size (all pi-score $<0.25 \&$ pi-score $>-0.25$ ) 
(2) "Ceiling effect": The two genes had positive effect sizes as single knock-outs and a negative pi-score, indicating the possibility that the double knock-out had hit a growth ceiling.

(3) "No deader-than-dead effect": The two genes had a negative effect size $(\mathrm{LFC}<-0.75)$ and a positive pi-score across all conditions, can't be "deader than dead".

We were left with a curated list of 9 gene-gene pairs that show interactions of potential interest for follow-up. We applied a z-score transformation to the pi-scores to correct for differences of growth rates between conditions leading to blunted pi-scores in conditions with slower growth rates. The resulting heatmap visually shows several gene-gene pairs have GxGxE interaction.

Hit validations For the follow-up experiments for single KOs, following spCas 9 sgRNAs were synthesized and cloned into the lentiCRISPR v2 vector (Genscript), and the resulting plasmids were used to generate the knockout lines.

SLC25A32 \#1 TAATGGGTTTGTAATGCAGA

SLC25A32 \#2 GGAGTAACCCCAAATATATG

SLC25A19 \#1 GGGTGCGCAGAACATCCACG

SLC25A19 \#2 GCTCCTATACATGGTCCCCA

EGFP control GGGCGAGGAGCTGTTCACCG

For the DKO cells, spCas9 guides for these genes were synthesized and cloned into either lentiCRISPR v2 vector (puromycin selection) and pXPR_BRD051 (hygromycin selection) vectors, and the resulting plasmids were used to generate the knockout lines.

SLC25A37\#1 GCACTCGGTCATGTACCCGG

SLC25A37\#2 AGCAATCAGCTGCATCCGGA

SLC25A39\#1 TGACCTCTACGCACCCATGG

SLC25A39\#2 GTGAAGATCGTGAGGCACGA

OR2M4-chr01 (OR2) CCATAAGGGACAGTTGACTG

OR11A1-chr06 (OR11) GTGATGCCAAAAATGCTGGA

For the study of SLC25A39, Lentiviral plasmids were used to ectopically express human SLC25A39WT, SLC25A39 ${ }^{\mathrm{D} 26 \mathrm{~A}}$, SLC25A39R225A/D226A and SLC25A39 ${ }^{\mathrm{K} 329 \mathrm{~A}}$ protein.

For rapid mitochondrial isolation experiments, following plasmids were used: pMXs-3XHAEGFP-OMP25 (Addgene plasmid \#83356) and pMXs-3XMyc-EGFP-OMP25 (Addgene plasmid \#83355).

To generate the DKOs and corresponding controls, cells were first infected with plasmids bearing the hygromycin selection cassette followed by selection with $0.25 \mathrm{mg} / \mathrm{ml}$ hygromycin (Roche, 10843555001) for one week, and then infected again with plasmids bearing puromycin selection cassette following by $2 \mu \mathrm{g} / \mathrm{ml}$ puromycin selection for two days. Two cutting controls were used here to control for the growth fitness defects caused by double-strand DNA breaks during the Cas9 cutting.

mRNA assays The mRNAs were isolated from 0.5 million cells using RNeasy Mini kit (Qiagen, 74104); the cDNA was prepared using SuperScript III First-Strand Synthesis SuperMix for qRT- 
PCR kit (Invitrogen, 11752050). qPCR experiment was performed on the QuantStudio 6 Flex Real-Time PCR Systems using the following Taqman probes: actin (Applied Biosystems, 4331182), GAPDH (Applied Biosystems, 402869), SLC25A37 (Applied Biosystems, 4331182) SLC25A39 (Applied Biosystems, 4448892)

Respiration assay Cellular respiration assays were performed using a seahorse XFe24 Analyzer (Agilent Technologies) coupled with BioTek Cytation 1 Cell Imager. 100k cells were plated in each well into a seahorse XFe24 culture plate that was pre-coated with cell-tak (Corning, 354240) working solution. Cellular OCR and ECAR were analyzed using the Mito Stress Test Kit (Agilent Technologies, 103015) at the following drug concentrations: $1.5 \mathrm{uM}$ oligomycin, $1 \mathrm{uM} \mathrm{FCCP}$ and $0.5 \mathrm{uM}$ mixture of rotenone and antimycin. At the end of the assay, the cells were stained with Hoechst dye (Thermo Fisher, 62249), and imaged with Cytation 1 for normalization.

LC-MS methods LC/MS-based analyses were performed on a Q Exactive plus benchtop orbitrap mass spectrometer equipped with an Ion Max source and a HESI II probe, which was coupled to a Dionex UltiMate 3000 UPLC system (Thermo Fisher Scientific) or a QE plus coupled to a Vanquish UHPLC.

Polar metabolite detection method was adapted with minor modification from previously literature $^{6,7}$. Cells were seeded at 2 million per well in 6 well plates for $24 \mathrm{hr}$ prior to harvesting. To prepare cellular metabolite extracts, transferred the cells and medium to $2 \mathrm{ml}$ tube, centrifuged at $600 \mathrm{~g}$ for $3 \mathrm{~min}$ at $4^{\circ} \mathrm{C}$, then aspirated the spent media and washed once with $1 \mathrm{ml}$ ice-cold PBS before lysis with acetonitrile-methanol-water (27:9:1 vol/vol/vol). For spent media metabolites, 50 $\mu \mathrm{l}$ spent media sample was aliquoted in the Eppendorf tube and mixed with $450 \mu \mathrm{L}$ ice-cold acetonitrile-methanol $(75: 25 \mathrm{vol} / \mathrm{vol})$. The extraction mixture was vortexed and centrifuged at $14,000 \mathrm{rpm}$ for $20 \mathrm{~min}$ at $4^{\circ} \mathrm{C}$, and $150 \mu \mathrm{l}$ supernatant was transferred into LC-MS glass vial for analysis.

Polar metabolites were analyzed on Xbrige BEH Amide XP HILIC Column, 100Å, $2.5 \mu$ m, 2.1 $\mathrm{mm}$ X $100 \mathrm{~mm}$ (Waters, 186006091) for chromatographic separation. The column oven temperature was $27^{\circ} \mathrm{C}$ and autosampler was $4^{\circ} \mathrm{C}$. Mobile phase A: $5 \%$ acetonitrile, $20 \mathrm{mM}$ ammonium acetate/ammonium hydroxide, $\mathrm{pH}$, and mobile phase $\mathrm{B}$ : $100 \%$ acetonitrile. LC gradient conditions at flow rate of $0.220 \mathrm{ml} / \mathrm{min}$ as following: $0 \mathrm{~min}: 85 \% \mathrm{~B}, 0.5 \mathrm{~min}: 85 \% \mathrm{~B}, 9$ min: $35 \%$ B, 11 min: $2 \% \mathrm{~B}, 13.5$ min: $85 \% \mathrm{~B}, 20 \mathrm{~min}: 85 \% \mathrm{~B}$.

The polar metabolites from mitochondria were analyzed on SeQuant ZIC-pHILIC polymeric $5 \mu \mathrm{m}$, 150x2.1 mm column (EMD-Millipore 150460). The LC-MS condition was same as described in nucleotides section.

The mass data were acquired in the polarity switching mode with full scan mode in a range of 70$1000 \mathrm{~m} / \mathrm{z}$, with the resolution at 70,000, the AGC target at $1 \mathrm{e}^{6}$, and the maximum injection time at $80 \mathrm{~ms}$, the sheath gas flow at 50 units, the auxiliary gas flow at 10 units, the sweep gas flow at 2 units, the spray voltage at $2.5 \mathrm{kV}$, the capillary temperature at $310^{\circ} \mathrm{C}$, and the auxiliary gas heater temperature at $370^{\circ} \mathrm{C}$. 
Compound discovery (Thermo Fisher Scientific) was used to pick peaks and integrate intensity from raw data. One filter was applied: max coefficient of variation within quality control samples $($ stdev $/$ mean $)<0.3$. The filtered metabolite lists were then annotated by searching against in-house chemical standard library with 5-ppm mass accuracy and 0.5 min retention time windows followed by manual curation. For the cellular profiling in the Supplementary Fig. 4f, consistently and significantly changed metabolites with $p$-value $<0.05$ that can be rescued are shown.

Folate species detection method was adapted with minor modification from previously literature ${ }^{8,9}$. Specifically, $\sim 2.5 \times 10^{6}$ cells were pelleted at room temperature, without washing, and extracted with dry ice cold $80 \%$ methanol containing $25 \mathrm{mM}$ sodium ascorbate, $10 \mathrm{mM}$ ammonium acetate. Cell extracts were vortexed and incubated on wet ice for 20 mins before centrifugation at 21,000 $\mathrm{g}$ for $20 \mathrm{~min}$ at $4^{\circ} \mathrm{C}$. Supernatants were dried down under nitrogen, re-suspended in $300 \mu 1$ resuspension buffer $\left(0.5 \%(\mathrm{w} / \mathrm{v})\right.$ ascorbic acid $+1 \%(\mathrm{w} / \mathrm{v}) \mathrm{K}_{2} \mathrm{HPO}_{4}+0.5 \%$ 2-mercaptoethanol). The polyglutamate tails of the folate species were cleaved by incubating the extracts with $30 \mu 1$ charcoal deactivated rat serum at $37^{\circ} \mathrm{C}$ for $1.5 \mathrm{hr}$ and adjusted to $\mathrm{pH} 4$ with $100 \mu \mathrm{l}$ of $1 \%$ formic acid. The charcoal deactivated rat serum was prepared by incubating $5 \mathrm{ml}$ rat serum (Sigma, R9759) with $250 \mathrm{mg}$ activated charcoal (Sigma, 9157) followed by end-to-end rotation at room temperature for $3 \mathrm{hr}$, the resulting serum were cleared by centrifugation and passing through $0.2 \mu \mathrm{m}$ filters. The treated samples were cleaned up using Agilent Bond Elute 96 square well plate (Agilent Technologies, A3961550). SPE wells were conditioned with $300 \mu 10.25 \%$ (w/v) ammonium acetate in $90 \%$ methanol, equilibrated with $300 \mu \mathrm{l} 0.25 \%$ ammonium acetate before sample loading, washed with $300 \mu 10.25 \%$ ammonium acetate and eluted with $300 \mu 10.25 \%$ ammonium acetate $+0.5 \%$ mercaptoethanol in 50\% methanol. Samples were dried down using nitrogen dryer, re-suspended in $75 \mu \mathrm{l}$ water before analysis, and $10 \mu \mathrm{l}$ sample were analyzed by LC-MS.

Folate species were analyzed on ACQUITY UPLC HSS T3 Column, 100A, $1.8 \mu \mathrm{m}, 2.1 \mathrm{~mm}$ X $100 \mathrm{~mm}$ (Waters, 186003539) for chromatographic separation. The column oven temperature was $30^{\circ} \mathrm{C}$ and autosampler was $4^{\circ} \mathrm{C}$. Mobile phase A: $20 \mathrm{mM}$ ammonium formate, $\mathrm{pH} 5$ (adjusted with formic acid), and mobile phase $\mathrm{B}$ : methanol. LC gradient conditions at flow rate of $0.250 \mathrm{ml} / \mathrm{min}$ as following: $0 \mathrm{~min}: 5 \% \mathrm{~B}, 3 \mathrm{~min}: 5 \% \mathrm{~B}, 11.4 \mathrm{~min}: 36 \% \mathrm{~B}, 11.5 \mathrm{~min}: 95 \% \mathrm{~B}, 14.0 \mathrm{~min}: 95 \% \mathrm{~B}$, $14.1 \mathrm{~min}: 5 \% \mathrm{~B}, 20 \mathrm{~min}: 5 \% \mathrm{~B}$.

The MS data were acquired in positive polarity in range of: $438-450 \mathrm{~m} / \mathrm{z}, 452-462 \mathrm{~m} / \mathrm{z}, 470-478$ $\mathrm{m} / \mathrm{z}$, resolution 70,000, AGC target $5 \mathrm{e}^{6}$ and maximum injection time of $300 \mathrm{~ms}$. MS parameters were: sheath gas flow 50, aux gas flow 10, sweep gas flow 2, spray voltage 2.50, Capillary temperature $310^{\circ} \mathrm{C}$, S-lens RF level -50 and aux gas heater temperature $370{ }^{\circ} \mathrm{C}$. Data acquisition was done using Xcalibur 4.1 (Thermo Scientific) and data analysis was done using the Tracefinder 4.1. with $5 \mathrm{ppm}$ mass tolerance. Retention time and MS/MS of folate species were matched with reference standards (supplementary Fig. 2f). The standards are folic acid (Sigma, F8758), THF (Schircks Laboratories, 16.207), 5,10-me ${ }^{+}$-THF (Schircks Laboratories, 16.230), 5,10-me-THF (Schircks Laboratories, 16.226), 5-methyl-THF (Schircks Laboratories, 16.235), 5-formyl-THF standards generated from $(6 \mathrm{R}, \mathrm{S})-5$-formyl-5,6,7,8-tetrahydropteroylpenta- $\gamma$-L-glutamic acid, lithium salt (Schircks Laboratories, 16.285).

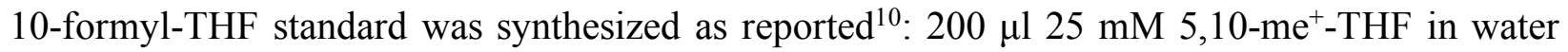
was added with $1 \mu \mathrm{l} 2$-mercaptoethanol and $5 \mu \mathrm{l} 2 \mathrm{~N} \mathrm{KOH}$ to a final $\sim \mathrm{pH} 8.5$, and incubated at 
room temperature for $30 \mathrm{~min}$. As 10-formyl-THF is very unstable, the synthesized standard was either analyzed immediately or stored at $-80^{\circ} \mathrm{C}$. Comparing to the MS/MS spectrum of the 10formyl-THF standard, the abovementioned extraction procedure might not preserve 10-formyl$\mathrm{THF}$ in the cellular extracts.

Purine intermediates and nucleotides detection was performed as previously described ${ }^{7,11}$. Specifically, for purine intermediates, approximately $2 \times 10^{6}$ cells were seeded at a density of $5 \times 10^{5}$ cells $/ \mathrm{ml}$ in corresponding media conditions and incubated overnight. The next day, equal number of cells in each condition were pelleted at $600 \mathrm{~g}$ for $5 \mathrm{~min}$ at room temperature, without washing, and extracted with $0.5 \mathrm{ml}$ dry ice-cold $80 \%$ methanol by vortex and incubation on wet ice for 20 min. The extracts were centrifuged at $21,000 \mathrm{~g}$ for $20 \mathrm{~min}$ at $4{ }^{\circ} \mathrm{C}$, and the supernatants were added

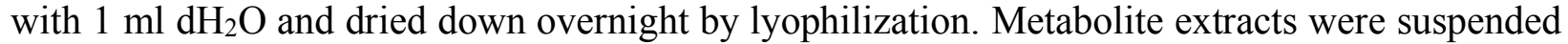
in $100 \mu \mathrm{l}$ of $60 \%$ acetonitrile, vortexed, and cleaned by centrifugation at 21,000 $\mathrm{g}$ for $10 \mathrm{~min}$ at $4{ }^{\circ} \mathrm{C}$. For NAD $(\mathrm{P})^{+}$and NAD(P)H quantification, cell pellets were lysed directly in $200 \mu \mathrm{l}$ ice-cold acetonitrile-methanol-water $(4: 4: 2)$ containing $0.1 \mathrm{M}$ formic acid containing $1 \mu \mathrm{M}$ of ${ }^{13} \mathrm{C}_{5} \mathrm{NAD}+$ and $1 \mu \mathrm{M}$ of d5-NADH as internal standards. Wash steps were omitted to avoid hydride exchange. Lysates were immediately neutralized with $17.5 \mu \mathrm{l}$ of $15 \%(\mathrm{w} / \mathrm{v})$ ammonium bicarbonate solution (after 2 minutes of lysis buffer addition), incubated on ice for $20 \mathrm{~min}$ and centrifuged at $21,000 \mathrm{~g}$ for $20 \mathrm{~min}$ at $4^{\circ} \mathrm{C}$. Supernatants were analyzed on the same day by the ZIC-pHILIC method mentioned below. Calibration curves of $\mathrm{NAD}^{+}, \mathrm{NADH}, \mathrm{NADP}^{+}$and $\mathrm{NADPH}$ were generated in extraction solvent. External calibration curve was used for the $\mathrm{NADP}^{+}$and $\mathrm{NADPH}$ quantification because of lack of respective isotope labeled standards.

Nucleotides were separated by SeQuant ZIC-pHILIC polymeric $5 \mu \mathrm{m}, 150 \times 2.1 \mathrm{~mm}$ column (EMD-Millipore 150460). Mobile phase A: $20 \mathrm{mM}$ ammonium carbonate in water, $\mathrm{pH} 9.6$ (adjusted with ammonium hydroxide), and mobile phase B: acetonitrile. The column was held at $27^{\circ} \mathrm{C}$, injection volume $5 \mu \mathrm{l}$, and an autosampler temperature of $4^{\circ} \mathrm{C}$. LC conditions at flow rate of $0.15 \mathrm{ml} / \mathrm{min}$ as following: $0 \mathrm{~min}: 80 \% \mathrm{~B}, 0.5 \mathrm{~min}: 80 \% \mathrm{~B}, 20.5 \mathrm{~min}: 20 \% \mathrm{~B}, 21.3 \mathrm{~min}: 20 \% \mathrm{~B}$, $21.5 \mathrm{~min}: 80 \%$ B till $29 \mathrm{~min}$. MS data were acquired with polarity switching mode. MS parameters were: sheath gas flow 30, aux gas flow 7, sweep gas flow 2, spray voltage 2.80 for negative and 3.80 for positive, capillary temperature $310^{\circ} \mathrm{C}$, S-lens RF level 50 and aux gas heater temp $370{ }^{\circ} \mathrm{C}$. Data acquisition was performed using Xcalibur 4.1 (Thermo Scientific) and performed in full scan mode with a range of 70-1000 m/z, resolution 70,000, AGC target $1 \mathrm{e}^{6}$ and maximum injection time of $80 \mathrm{~ms}$. Data analysis was done using Xcalibur 4.1 (Thermo Scientific) and the quality of integration for each chromatographic peak was reviewed. Metabolite annotation was done base on accurate mass $( \pm 5 \mathrm{ppm})$ and matching retention time as well as MS/MS fragmentation pattern from the pooled QC sample against in-house retention time + MS/MS library of reference standards. Metabolites which have $\mathrm{CV}<20 \%$ in pooled QC were used for the statistical analysis.

\section{Sequence analysis and structural modeling}

SLC25A39 orthologs in eukaryotic organisms were identified as Bidirectional best hits (BBH) from either protein BLAST or tblastn search. The sequences were aligned using MUSCLE (MUltiple Sequence Comparison by Log-Expectation) and the secondary structure annotated using ESPript. 
A homology model of human SLC25A39 was predicted using the SWISS-MODEL server based on the ADP/ATP ANT transporter c-state conformation (PDB: 1OKC). The putative substrate binding residues were predicted based on the previous structural and structure-function analysis ${ }^{12-}$ 15 .

\section{Mitochondrial isolation and uptake assay}

A rapid mitochondria immunoisolation method was adopted as previously described ${ }^{16,17}$. Specifically, approximately 30 million cell lines expressing mitoIP constructs were washed with PBS and collected at $1,000 \mathrm{~g}$ for $2 \mathrm{~min}$ at $4{ }^{\circ} \mathrm{C}$, suspended in $1 \mathrm{ml} \mathrm{KPBS}$ buffer $(136 \mathrm{mM} \mathrm{KCl}, 10$ $\left.\mathrm{mM} \mathrm{KH} \mathrm{PO}_{4}, \mathrm{pH} 7.25\right)$ to homogenous single cell suspension, and lysed by 20 gentle strokes in the $2 \mathrm{ml}$ hand-held Dounce tissue grinder with tight-fitting pestle B (VMR, Kontes). Cell lysates were cleared by centrifugation at $1,000 \mathrm{~g}$ for $2 \mathrm{~min}$ at $4{ }^{\circ} \mathrm{C}$, and transferred to a new $1.5 \mathrm{ml} \mathrm{EP}$ tube containing $100 \mu \mathrm{l}$ pre-washed anti-HA magnetic beads (Pierce, 88837). After an end-to-end rotator incubation for $3.5 \mathrm{~min}$ in the cold room, the isolated mitochondria were washed with KPBS buffer for 3 times. For the metabolite profiling experiment, mitochondrial metabolites were extracted with $50 \mu \mathrm{l}$ acetonitrile-methanol-water (27:9:1 vol/vol/vol). For western blotting experiment, mitochondrial proteins were lysed with $100 \mu 1$ RIPA lysis buffer.

Mitochondrial uptake assay The isolated mitochondria were incubated with $0.2 \mathrm{mM}$ labeled GSH, GSH-[glycine-13C2, 15N] (Sigma, 683620), in $100 \mu \mathrm{l}$ assay buffer (110 mM sucrose, $20 \mathrm{mM}$ HEPES pH7.4, $10 \mathrm{mM} \mathrm{KH}_{2} \mathrm{PO}_{4}, 3 \mathrm{mM} \mathrm{MgCl} 2,1 \mathrm{mM}$ EGTA, 0.1\% BSA). The uptake assay is performed as room temperature by a horizontal shaking at $170 \mathrm{rpm}$ and manually mix every 10 min. At indicated time point, an aliquot of isolated mitochondria suspension was taken and washed three times with KPBS buffer, and metabolite extracted with $50 \mu 1$ ice acetonitrile-methanol-water (27:9:1 vol/vol/vol) for immediate LC-MS analysis.

\section{Western blotting}

Following antibodies and concentration were used: Anti-SLC25A39 (Proteintech, 14963-1-AP, 1:100); Anti-VDAC (Cell Signaling Technology, 4661T, 1:1000); Anti-SHMT2 (Sigma-Aldrich, HPA-020549, 1:1000); Anti-Calreticulin (Cell Signaling Technology, 12238T, 1:1000); AntiLAMP2 (Santa Cruz Biotechnology, sc-18822, 1:1000).

\section{Statistics analysis and data presentation}

For cell characterization and metabolite profiling experiments, data are shown as mean \pm s.d., $n \geq 3$ biologically independent samples unless stated otherwise. Statistical significance was calculated using two-tailed $t$-test. Significance level were indicated as $* * * p<0.001, * * p<0.01, * p<0.05$ and n.s. $p>0.05$. The screens were performed in two batches and the results were validated in the follow-up studies. All the other experiments were validated at least two times. Statistical analysis was performed using GraphPad Prism 7.0a, or as reported by the relevant computational tools.

\section{Data availability}

The screen data are available within the paper and its supplementary table files. Other data supporting the findings of this study are available from the corresponding authors upon request.

\section{Code availability}


The code for analyzing the screening data uses standard libraries and techniques that have been reported previously in the literature. The code can be made available from the corresponding author upon reasonable request.

1. Najm, F. J. et al. Orthologous CRISPR-Cas9 enzymes for combinatorial genetic screens. Nat Biotechnol 36, 179-189 (2018).

2. Calvo, S. E., Clauser, K. R. \& Mootha, V. K. MitoCarta2.0: an updated inventory of mammalian mitochondrial proteins. Nucleic Acids Res 44, D1251-1257 (2016).

3. Arroyo, J. D. et al. A Genome-wide CRISPR Death Screen Identifies Genes Essential for Oxidative Phosphorylation. Cell Metab 24, 875-885 (2016).

4. Aguirre, A. J. et al. Genomic Copy Number Dictates a Gene-Independent Cell Response to CRISPR/Cas9 Targeting. Cancer Discov 6, 914-929 (2016).

5. Fischer, B. et al. A map of directional genetic interactions in a metazoan cell. Elife 4 (2015).

6. Shen, H. et al. The Human Knockout Gene CLYBL Connects Itaconate to Vitamin B12. Cell 171, 771-782 e711 (2017).

7. Wang, L. W. et al. Epstein-Barr-Virus-Induced One-Carbon Metabolism Drives B Cell Transformation. Cell Metab 30, 539-555 e511 (2019).

8. Kanarek, N. et al. Histidine catabolism is a major determinant of methotrexate sensitivity. Nature 559, 632-636 (2018).

9. Chen, L. et al. NADPH production by the oxidative pentose-phosphate pathway supports folate metabolism. Nature Metabolism 1, 404-415 (2019).

10. Stover, P. \& Schirch, V. Synthesis of (6S)-5-formyltetrahydropteroyl-polyglutamates and interconversion to other reduced pteroylpolyglutamate derivatives. Anal Biochem 202, 82-88 (1992).

11. Lu, W., Wang, L., Chen, L., Hui, S. \& Rabinowitz, J. D. Extraction and Quantitation of Nicotinamide Adenine Dinucleotide Redox Cofactors. Antioxid Redox Signal 28, 167 179 (2018).

12. Kunji, E. R. \& Robinson, A. J. The conserved substrate binding site of mitochondrial carriers. Biochim Biophys Acta 1757, 1237-1248 (2006).

13. Monne, M. et al. Substrate specificity of the two mitochondrial ornithine carriers can be swapped by single mutation in substrate binding site. J Biol Chem 287, 7925-7934 (2012).

14. Robinson, A. J. \& Kunji, E. R. Mitochondrial carriers in the cytoplasmic state have a common substrate binding site. Proc Natl Acad Sci U S A 103, 2617-2622 (2006).

15. Ruprecht, J. J. et al. The Molecular Mechanism of Transport by the Mitochondrial ADP/ATP Carrier. Cell 176, 435-447 e415 (2019).

16. Chen, W. W., Freinkman, E. \& Sabatini, D. M. Rapid immunopurification of mitochondria for metabolite profiling and absolute quantification of matrix metabolites. Nat Protoc $\mathbf{1 2}$, 2215-2231 (2017).

17. Chen, W. W., Freinkman, E., Wang, T., Birsoy, K. \& Sabatini, D. M. Absolute Quantificationof Matrix Metabolites Reveals the Dynamics of Mitochondrial Metabolism. Cell 166, 1324-1337 (2016) 